\title{
Construction of Isochromenes via Ruthenium-Catalyzed Reaction of Oxabenzonorbornenes with Propargylic Alcohols
}

\author{
Karine Villeneuve and William Tam* \\ Guelph-Waterloo Centre for Graduate Work in Chemistry and Biochemistry, \\ Department of Chemistry, University of Guelph, Guelph, Ontario, Canada N1G 2W1
}

\section{Supporting Information}

\section{Contents}

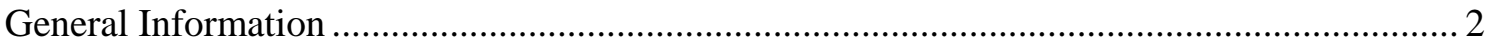

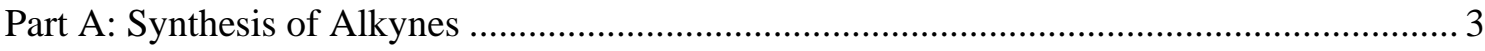

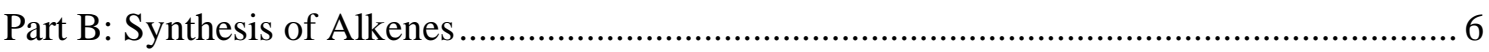

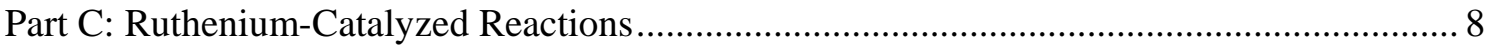




\section{General Information}

All reactions were carried out in an atmosphere of dry nitrogen at ambient temperature unless otherwise stated. Standard column chromatography was performed on 230-400 mesh silica gel (obtained from Silicycle) using flash column chromatography techniques. ${ }^{1}$ Analytical thin-layer chromatography (TLC) was performed on Silicycle precoated silica gel $F_{254}$ plates. All glassware was flame dried under an inert atmosphere of dry nitrogen. Infrared spectra were taken on a Bomem MB-100 FTIR spectrophotometer. ${ }^{1} \mathrm{H}$ and ${ }^{13} \mathrm{C}$ NMR spectra were recorded on a Bruker Avance-300 and 400 spectrometers. Chemical shifts for ${ }^{1} \mathrm{H}$ NMR spectra are reported in parts per million (ppm) from tetramethylsilane with the solvent resonance as the internal standard (chloroform: $\delta 7.26 \mathrm{ppm}$ ). Chemical shifts for ${ }^{13} \mathrm{C}$ NMR spectra are reported in parts per million (ppm) from tetramethylsilane with the solvent as the internal standard (deuterochloroform: $\delta 77.0 \mathrm{ppm}$ ). Chemical shifts for ${ }^{2} \mathrm{H}$ NMR spectra are reported in parts per million (ppm) from tetramethylsilane with the solvent as the internal standard (deuterochloroform: $\delta 7.26 \mathrm{ppm}$ ). High resolution mass spectra were done by McMaster Regional Centre for Mass Spectrometry at McMaster University, Hamilton, Ontario. Elemental analyses were performed by Canadian Microanalytical Service Ltd., British Columbia or by Quantitative Technologies Inc., New Jersey.

Reagents: Unless stated otherwise, commercial reagents were used without purification. Solvents were purified by distillation under dry nitrogen: from $\mathrm{CaH}_{2}$ (1,2-dichloroethane, hexanes, DMF, pyridine); from sodium (toluene); from magnesium/iodine ( $\mathrm{MeOH},{ }^{\mathrm{i}} \mathrm{PrOH}$, ${ }^{n} \mathrm{PrOH}, \mathrm{EtOH},{ }^{\mathrm{n}} \mathrm{BuOH}$ ) and from potassium/benzophenone (THF). Alkene $2 \mathbf{f}$ was bought from Sigma-Aldrich Co. and used without further purification. [Cp* $\left.\mathrm{Ru}\left(\mathrm{CH}_{3} \mathrm{CN}\right)\right] \mathrm{PF}_{6}$ was bought from Strem Chemicals. Alkynes $\mathbf{4 a},{ }^{2} \mathbf{4 a}-d,{ }^{3} \mathbf{4} \mathbf{b},{ }^{3} \mathbf{4} \mathbf{c},{ }^{3} \mathbf{4 g},{ }^{4} \mathbf{4 j},{ }^{3}$ bicyclic alkenes $\mathbf{2 a},{ }^{5}$ $\mathbf{2 b},{ }^{6} \mathbf{2 d}{ }^{7} \mathbf{2 e},{ }^{8} \mathbf{2 g},{ }^{5} \mathbf{2 g}^{9}$ and $\mathrm{Cp} * \mathrm{Ru}(\mathrm{COD}) \mathrm{Cl}^{10}$ were prepared according to literature procedures.

\footnotetext{
${ }^{1}$ W. C. Still, M. Kahn, A. Mitra, J. Org. Chem. 1978, 43, 2923.

${ }^{2}$ Villeneuve, K.; Jordan, R. W.; Tam, W. Synlett 2003, 2123.

${ }^{3}$ Villeneuve, K.; Tam, W. Organometallics 2006, 25, 843.

${ }^{4}$ Zhang, X.; Sarkar, S.; Larock, R. C. J. Org. Chem. 2006, 71, 236.

${ }^{5}$ Nakayama, J.; Sakai, A.; Hoshimo, M. J. Org. Chem. 1984, 49, 5084.

${ }^{6}$ Giles, R. G. F.; Hughes, A. B.; Sargent, M. V. J. Chem. Soc., Perkin Trans. 1 1991, 1581.

${ }^{7}$ Lu, S.Y.; Quayle, P.; Heatley, F.; Booth, C.; Yeates, S. G.; Padget, J. C. Macromolecules 1992, $25,2692$.

${ }^{8}$ Stork, G.; Tamelen, V.; Eugene, E. E.; Friedman, L. J.; Burgstahler, A. W. J. Am. Chem. Soc. 1953, 75, 384.

${ }^{9}$ Best, W. M.; Collins, P. A.; McCulloch, R. K.; Wege, D. Aust. J. Chem. 1982, 35, 843.
} 


\section{Part A: Synthesis of Alkynes}

General procedure (A) for the preparation of alkynes. To a cold solution $\left(-70{ }^{\circ} \mathrm{C}\right)$ of hexamethyldisilazane (HMDS, 1.2 eq.) in THF (0.6 M versus ethyl propiolate) was added dropwise a solution of butyllithium in hexanes (BuLi, $2.5 \mathrm{M}, 1.2$ eq.). The reaction was stirred for 15 min then ethyl propiolate (1.0 eq.) was added dropwise. The resulting mixture was allowed to stir at $-70^{\circ} \mathrm{C}$ for 20 min prior to add the electrophile (1.4 eq.). The reaction was monitored by TLC and upon completion, quenched with water and then allowed to warm to room temperature. The reaction mixture was diluted with ethyl acetate the layers were separated. The aqueous layer was extracted three times with ethyl acetate, and the combined organic extracts were then washed with brine, dried over anhydrous magnesium sulfate, filtrated and concentrated to dryness. The crude product was the purified by column chromatography.

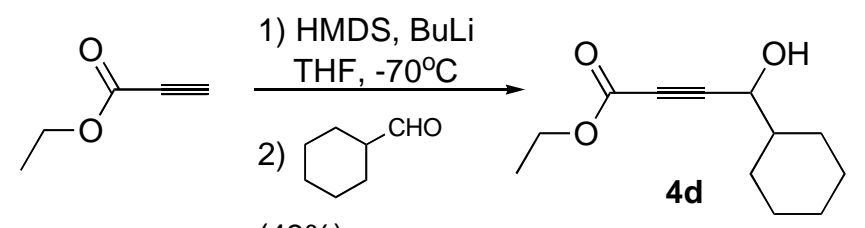

$(43 \%)$

Following the above general procedure A with HMDS (0.75 mL, $3.6 \mathrm{mmol})$, THF (5.0 mL), BuLi $(1.40 \mathrm{~mL}, \quad 3.54 \mathrm{mmol})$, ethyl propiolate $(0.30 \mathrm{~mL}, 3.0 \mathrm{mmol})$ and cyclohexylcarboxaldehyde $(0.60 \mathrm{~mL}, 4.0 \mathrm{mmol})$. The crude product was purified by column chromatography (gradient EtOAc:hexanes = 1: 9 to 3:7) to give 4d (0.2697 g, $1.283 \mathrm{mmol}$, $43 \%$ ) as a colorless oil. $\mathrm{R}_{\mathrm{f}} 0.23$ (EtOAc:hexanes = 1:9); IR (neat) 3417, 2930, 2855, 2234, 1714, 1630, 1451, $1241 \mathrm{~cm}^{-1} ;{ }^{1} \mathrm{H} \mathrm{NMR}\left(\mathrm{CDCl}_{3}, 400 \mathrm{MHz}\right) \delta 4.26$ (app t, $1 \mathrm{H}, J=6.1 \mathrm{~Hz}$ ), 4.22 (q, 2H, $J=7.1 \mathrm{~Hz}), 2.44$ (d, 1H, $J=6.0 \mathrm{~Hz}), 1.84-1.87$ (m, 2H), 1.75-1.78 (m, 2H), 1.581.68 (m, 2H), 1.30 (t, 3H, $J=7.1 \mathrm{~Hz}), 1.00-1.26$ (m, 5H); ${ }^{13} \mathrm{C}$ NMR (APT, $\mathrm{CDCl}_{3}, 100 \mathrm{MHz}$ ) $\delta$ 153.5, 87.2, 77.3, 66.8, 62.1, 43.6, 28.3, 28.1, 26.1, 25.7, 13.9; HRMS (CI) calcd. for $\mathrm{C}_{12} \mathrm{H}_{18} \mathrm{O}_{3}\left((\mathrm{M}+\mathrm{H})^{+}\right)$: 211.1336; found: 211.1334 .

\footnotetext{
${ }^{10}$ P. J. Fagan, W. S. Mahoney , J. C. Calabrese, I. D. Williams, Organometallics 1990, 9, 1843.
} 


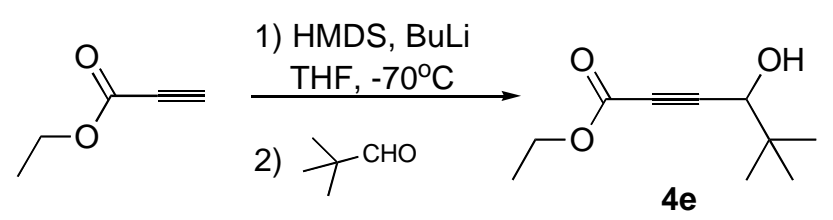

$(55 \%)$

Following the above general procedure A with HMDS (0.75 mL, $3.6 \mathrm{mmol})$, THF (5.0 mL), BuLi (1.40 mL, $3.54 \mathrm{mmol})$, ethyl propiolate $(0.30 \mathrm{~mL}, 3.0 \mathrm{mmol})$ and trimethylacetaldehyde (0.45 mL, $4.1 \mathrm{mmol})$. The crude product was purified by column chromatography (gradient EtOAc:hexanes $=1: 9$ to $1: 4)$ to give $4 \mathbf{e}(0.2985 \mathrm{~g}, 1.620 \mathrm{mmol}, 55 \%)$ as a colorless oil. $\mathrm{R}_{\mathrm{f}}$ 0.21 (EtOAc:hexanes = 1:9); IR (neat) 3457, 2967, 2872, 2234, 1716, $1254 \mathrm{~cm}^{-1}$; ${ }^{1} \mathrm{H}$ NMR $\left(\mathrm{CDCl}_{3}, 400 \mathrm{MHz}\right) \delta 4.22$ (q, 2H, $\left.J=7.1 \mathrm{~Hz}\right), 4.12$ (s, 1H), 2.36 (br s, 1H), 1.30 (t, 3H, $J=$ 7.1 Hz), 1.01 (s, 9H); ${ }^{13} \mathrm{C}$ NMR (APT, $\mathrm{CDCl}_{3}, 100 \mathrm{MHz}$ ) $\delta$ 153.4, 86.9, 77.5, 71.0, 62.1, 36.0, 26.2, 25.2, 14.0; HRMS (CI) calcd. for $\mathrm{C}_{10} \mathrm{H}_{16} \mathrm{O}_{3}\left((\mathrm{M}+\mathrm{H})^{+}\right)$: 185.1178; found: 185.1184.

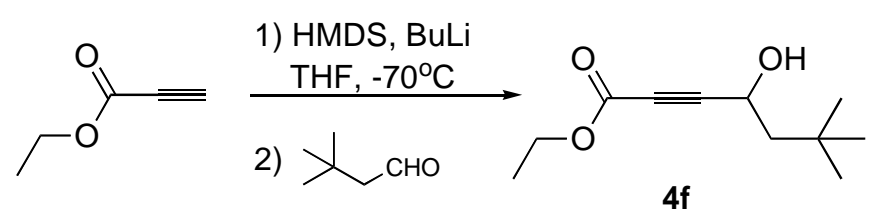

$(47 \%)$

Following the above general procedure A with HMDS (0.75 mL, $3.6 \mathrm{mmol})$, THF (5.0 mL), BuLi (1.40 mL, $3.54 \mathrm{mmol})$, ethyl propiolate $(0.30 \mathrm{~mL}, 3.0 \mathrm{mmol})$ and 3,3dimethylbutaldehyde $(0.50 \mathrm{~mL}, 4.0 \mathrm{mmol})$. The crude product was purified by column chromatography (gradient EtOAc:hexanes = 1:19 to 1:4) to give $\mathbf{4 f}(0.2768 \mathrm{~g}, 1.396 \mathrm{mmol}$, $47 \%$ ) as a colorless oil. $\mathrm{R}_{\mathrm{f}} 0.26$ (EtOAc:hexanes = 1:9); IR (neat) 3432, 2956, 2907, 2871, 2235, 1714, $1245 \mathrm{~cm}^{-1}$; ${ }^{1} \mathrm{H}$ NMR $\left(\mathrm{CDCl}_{3}, 400 \mathrm{MHz}\right) \delta 4.55-4.60$ (m, 1H), 4.22 (q, 2H, $J=$ $7.1 \mathrm{~Hz}), 2.05$ (m, 1H), 2.01-2.08 (m, 2H), 1.30 (t, 3H, $J=7.1 \mathrm{~Hz}), 0.98$ (s, 9H); ${ }^{13} \mathrm{C} \mathrm{NMR}$ $\left(\mathrm{APT}, \mathrm{CDCl}_{3}, 100 \mathrm{MHz}\right) \delta 153.5,88.8,76.4,62.1,59.9$, 50.5, 30.2, 29.8, 14.0; HRMS (EI) calcd. for $\mathrm{C}_{11} \mathrm{H}_{18} \mathrm{O}_{3}\left(\mathrm{M}^{+}\right)$: 198.1256; found: 198.1262 . 


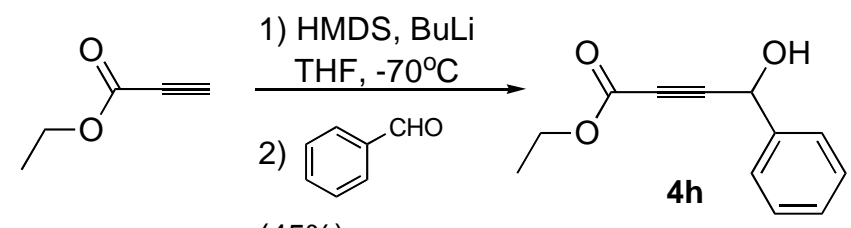

$(45 \%)$

Following the above general procedure A with HMDS (1.25 mL, $5.90 \mathrm{mmol})$, THF (6.0 mL), BuLi (2.40 mL, $6.00 \mathrm{mmol})$, ethyl propiolate $(0.50 \mathrm{~mL}, 4.9 \mathrm{mmol})$ and benzaldehyde $(0.60$ $\mathrm{mL}, 5.9 \mathrm{mmol}$ ). The crude product was purified by column chromatography (gradient EtOAc:hexanes $=1: 19$ to $1: 4)$ to give $\mathbf{4 h}(0.4520 \mathrm{~g}, 2.213 \mathrm{mmol}, 45 \%)$ as a colorless oil. $\mathrm{R}_{\mathrm{f}}$ 0.41 (EtOAc:hexanes = 1:4); IR (neat) 3411, 2984, 223, 1713, $1251 \mathrm{~cm}^{-1} ;{ }^{1} \mathrm{H}$ NMR $\left(\mathrm{CDCl}_{3}\right.$, $400 \mathrm{MHz}) \delta 7.52$ (dd, 2H, $J=7.8,1.4 \mathrm{~Hz}), 7.36-7.42(\mathrm{~m}, 3 \mathrm{H}), 5.56$ (s, 1H), 4.24 (q, 2H, $J=$ $7.1 \mathrm{~Hz}$ ), 2.72 (br s, 1H), 1.31 (t, 3H, $J=7.1 \mathrm{~Hz}$ ); ${ }^{13} \mathrm{C}$ NMR (APT, $\left.\mathrm{CDCl}_{3}, 100 \mathrm{MHz}\right) \delta 153.3$, 138.5, 128.9, 128.8, 126.6, 86.0, 77.9, 64.3, 62.3, 13.9; HRMS (CI) calcd. for $\mathrm{C}_{12} \mathrm{H}_{12} \mathrm{O}_{3}$ $\left((\mathrm{M}+\mathrm{H})^{+}\right): 205.0865$; found: 205.0860 .

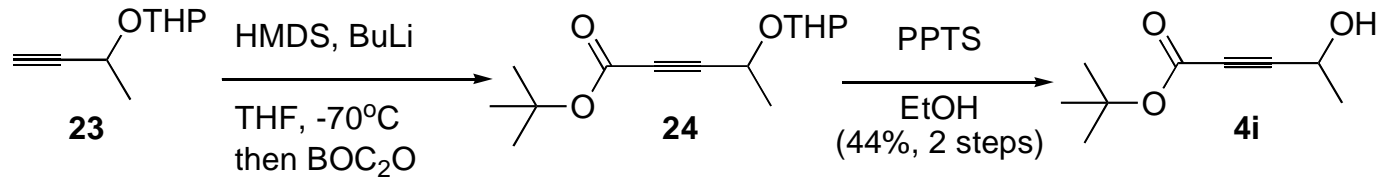

To a cold solution $\left(-70^{\circ} \mathrm{C}\right)$ of hexamethyldisilazane (HMDS, $\left.0.66 \mathrm{~mL}, 3.1 \mathrm{mmol}\right)$ in THF (3.5 mL) was added dropwise a solution of butyllithium in hexanes (2.5M, $1.2 \mathrm{~mL}, 3.0$ mmol). The reaction was stirred for 15 min then a solution of 23 (0.4019 g, $2.606 \mathrm{mmol})$ in THF $(1.0 \mathrm{~mL})$ was added via cannula. The resulting mixture was allowed to stir at $-70{ }^{\circ} \mathrm{C}$ for 20 min prior to be added to a cold $\left(-70{ }^{\circ} \mathrm{C}\right)$ solution of di-tert-butyldicarbonate $(0.7462 \mathrm{~g}$, $3.419 \mathrm{mmol}$ ) in THF (3.0 mL). The reaction was stirred at $-70{ }^{\circ} \mathrm{C}$ for $20 \mathrm{~min}$ then allowed to warm up to $-30{ }^{\circ} \mathrm{C}$ over $1 \mathrm{~h}$. Upon completion, the reaction mixture was quenched with water and then diluted with diethyl acetate. The layers were separated. The aqueous layer was extracted three times with ethyl acetate, and the combined organic extracts were washed with brine, dried over anhydrous magnesium sulfate, filtrated and concentrated to dryness. The crude product was the purified by column chromatography (gradient EtOAc:hexanes $=1: 9$ to 1:4). The THP group was then removed by refluxing 24 in ethanol (15 $\mathrm{mL})$ in presence of pyridinium para-toluenesulfonate $(80.2 \mathrm{mg}, 0.319 \mathrm{mmol})$ for $3 \mathrm{~h}$. The solvent was evaporated under reduced pressure, and the crude product was purified by column chromatography (gradient $\mathrm{Et}_{2} \mathrm{O}$ :hexanes = 1:9 to 2:3) to give $\mathbf{4 i}(0.1418 \mathrm{~g}, 0.8380 \mathrm{mmol}, 44 \%)$ as a colorless oil. $\mathrm{R}_{\mathrm{f}} 0.26$ (EtOAc:hexanes = 1:9); IR (neat) 3411, 2984, 2937, 2875, 2231,1708, $1278 \mathrm{~cm}^{-1}$; 
${ }^{1} \mathrm{H}$ NMR $\left(\mathrm{CDCl}_{3}, 400 \mathrm{MHz}\right) \delta 4.60$ (m, 1H), 2.97 (br d, 1H, $\left.J=5.2 \mathrm{~Hz}\right), 1.474$ (d, 3H, $J=6.9$

$\mathrm{Hz}$ ), 1.465 (s, 9H); ${ }^{13} \mathrm{C}$ NMR (APT, $\left.\mathrm{CDCl}_{3}, 100 \mathrm{MHz}\right) \delta$ 152.6, 86.3, 83.7, 76.7, 57.8, 27.9, 23.2; HRMS (CI) calcd. for $\mathrm{C}_{9} \mathrm{H}_{14} \mathrm{O}_{3}\left((\mathrm{M}+\mathrm{H})^{+}\right)$: 171.1021; found: 171.1027 .

\section{Part B: Synthesis of Alkenes}

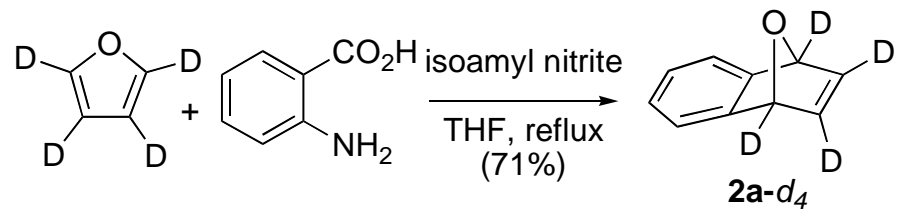

Alkene $2 \mathbf{a}-\boldsymbol{d}_{4}$ : To a stirred solution of isoamyl nitrite $(0.90 \mathrm{~mL}, 6.7 \mathrm{mmol})$ and furan- $\mathrm{d}_{4}(1.0$ g, $14 \mathrm{mmol})$ in THF (6 mL) under nitrogen was added a solution of anthranilic acid (0.380 g, $2.77 \mathrm{mmol}$ ) in THF ( $3 \mathrm{~mL}$ ) through an addition funnel. The addition was done over 45min, starting at room temperature then gently refluxing after $30 \mathrm{~min}$. The resulting red solution was further refluxed for another hour and allowed to cool to room temperature. The reaction mixture was partitioned between ethyl acetate and aqueous $5 \% \mathrm{NaHCO}_{3}$ solution, and the layers were separated. The organic extract was washed with another portion of aqueous $5 \%$ $\mathrm{NaHCO}_{3}$, then with water followed by brine, prior to be dried over $\mathrm{MgSO}_{4}$ and concentrated under reduce pressure. The crude product was purified by column chromatography (1:9 EtOAc:hexanes) to provide 2a- $d_{4}(0.291 \mathrm{~g}, 1.96 \mathrm{mmol}, 71 \%) .{ }^{1} \mathrm{H}$ NMR $\left(\mathrm{CDCl}_{3}, 300 \mathrm{MHz}\right) \delta$ $7.23(\mathrm{dd}, 2 \mathrm{H}, J=5.0,3.0 \mathrm{~Hz}), 6.94$ (dd, $2 \mathrm{H}, J=5.0,3.0 \mathrm{~Hz}) ;{ }^{13} \mathrm{C} \mathrm{NMR}\left(\mathrm{APT}, \mathrm{CDCl}_{3}, 75\right.$ MHz) $\delta$ 148.8, $142.3(\mathrm{t}, J=28 \mathrm{~Hz}), 124.7,120.0,81.6(\mathrm{t}, J=25 \mathrm{~Hz}) ;{ }^{2} \mathrm{H} \mathrm{NMR}\left(\mathrm{CHCl}_{3}, 61\right.$ MHz) $\delta$ 7.10, 5.74. HRMS (CI) calcd. for $\mathrm{C}_{10} \mathrm{H}_{4}{ }^{2} \mathrm{H}_{4} \mathrm{O}\left((\mathrm{M}+\mathrm{H})^{+}\right)$: 149.0904; found: 149.0906.

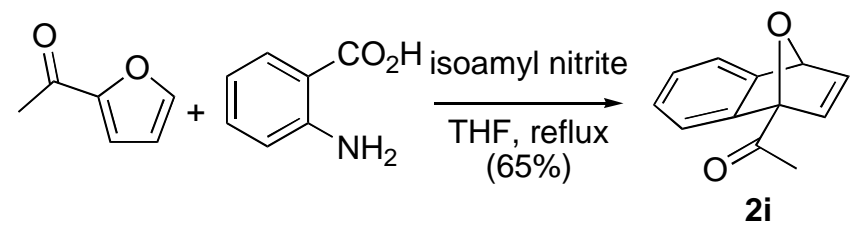

Alkene 2i. To a stirring solution of isoamyl nitrite $(14.5 \mathrm{~mL}, 109 \mathrm{mmol})$ and 2-acetylfuran (7.3 ml, $73 \mathrm{mmol})$ in dry THF $(100 \mathrm{~mL})$ under $\mathrm{N}_{2}$, a solution of anthranilic acid (5.0 g, 36.5 mmol) in dry THF (25 mL) was added slowly by drop funnel. The solution was warmed up to $65-70{ }^{\circ} \mathrm{C}$ during the addition of anthranilic acid and refluxed for 2 hrs following the addition. The resulting mixture was concentrated and purified by column chromatography 
(EtOAc:hexanes = 1.5:8.5) to afford alkene $2 \mathbf{i}$ as a pale yellow oil (4.57 g, $24.5 \mathrm{mmol}, 65 \%$ ). $R_{f} 0.53$ (EtOAc:hexanes = 3:7); IR (neat) 3070, 3016, 1715, 1454, 1441, 1417, 1365, 1320, 1274, $1234 \mathrm{~cm}^{-1}$; ${ }^{1} \mathrm{H}$ NMR $\left(\mathrm{CDCl}_{3}, 400 \mathrm{MHz}\right) \delta 7.26$ (m, 2H), 7.04 (m, 4H), 5.82 (br. s, 1H), 2.42 (br. s, 3H); ${ }^{13} \mathrm{C}$ NMR (APT, $\left.\mathrm{CDCl}_{3}, 100 \mathrm{MHz}\right) \delta$ 205.2, 148.0, 147.5, 143.4, 142.3, 125.6, 125.2, 120.6, 119.5, 114.5, 95.7, 82.3, 26.8. HRMS (EI) calcd. for $\mathrm{C}_{12} \mathrm{H}_{10} \mathrm{O}_{2}\left(\mathrm{M}^{+}\right)$: 186.0681; found: 186.0688 .

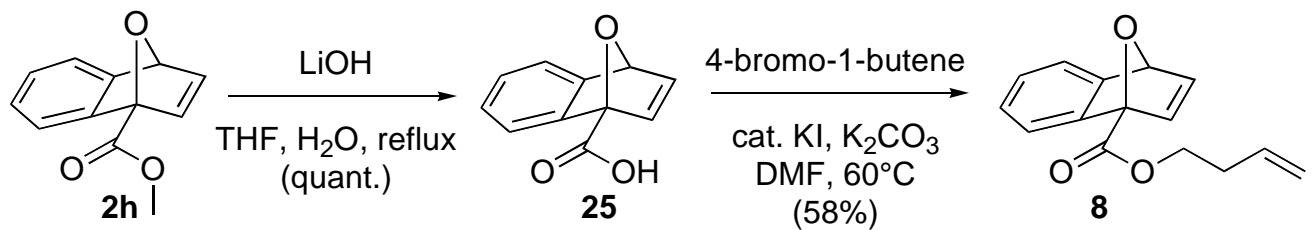

In a round-bottomed flask equipped with a condenser were successively loaded $\mathbf{2 h}(0.5012 \mathrm{~g}$, $2.479 \mathrm{mmol})$, THF (7.5 mL), $\mathrm{H}_{2} \mathrm{O}(2.5 \mathrm{~mL})$ and $\mathrm{LiOH}(1.24 \mathrm{~g}, 29.6 \mathrm{mmol})$. The biphasic reaction mixture was allowed to gently refluxed for $30 \mathrm{~min}$, prior to be cooled to $0{ }^{\circ} \mathrm{C}$. The mixture was acidified with conc. $\mathrm{H}_{2} \mathrm{SO}_{4}$ to ca. $\mathrm{pH}=3$. The solid was filtrated and washed with cold water, then dissolved in EtOAc, dried over magnesium sulfate and concentrated to dryness to give 25 as a beige solid (0.4632 g, $2.461 \mathrm{mmol}$, quantitative yield). ${ }^{1} \mathrm{H}$ NMR $\left(\mathrm{CDCl}_{3}, 400 \mathrm{MHz}\right) \delta$ 7.43-7.47 (m, 1H), 7.28-7.31 (m, 1H), 7.01-7.14 (m, 4H), 5.87 (d, 1H, $J$ $=1.5 \mathrm{~Hz}) ;{ }^{13} \mathrm{C}$ NMR $\left(\mathrm{APT}, \mathrm{CDCl}_{3}, 100 \mathrm{MHz}\right) \delta 172.7,147.4,146.6,143.7,142.1,125.9$, 125.4, 120.7, 120.0, 90.1, 82.6. HRMS (CI) calcd. for $\mathrm{C}_{11} \mathrm{H}_{8} \mathrm{O}_{3}\left((\mathrm{M}+\mathrm{H})^{+}\right)$: 189.0552; found: 189.0560. Acid 25 was used for the next step without further purification.

To a mixture of 25 (0.1528 g, $0.8077 \mathrm{mmol})$ and $\mathrm{K}_{2} \mathrm{CO}_{3}(0.1812 \mathrm{~g}, 1.311 \mathrm{mmol})$ in DMF (3.0 $\mathrm{mL}$ ) was added potassium iodide (ca. $10 \mathrm{mg}$ ). The flask was then equipped with a condenser and purged with nitrogen prior to slowly add 4-bromo-1-butene $(0.40 \mathrm{~mL}, 3.9 \mathrm{mmol})$. The reaction was heated at $60^{\circ} \mathrm{C}$ for $24 \mathrm{~h}$ then allowed to cool to room temperature. The mixture was partitioned between $1 \mathrm{~N}$ aqueous $\mathrm{HCl}$ and diethyl ether. Layers were separated; aqueous phase was extracted 3 times with diethyl ether. The combined organic layers were washed with brine, dried over $\mathrm{MgSO}_{4}$ and concentrated under reduced pressure. The crude product was purified by column chromatography (gradient EtOAc:hexanes $=1: 19$ to 1:9) to provide 8 (0.1123g, $0.4635 \mathrm{mmol}, 58 \%)$ as a pale yellow oil. : $R_{f} 0.29$ (EtOAc:hexanes=1:4); IR $\left(\mathrm{CH}_{2} \mathrm{Cl}_{2}\right)$ 3134, 3076, 3052, 3015, 2980, 2961, 2902, $1737 \mathrm{~cm}^{-1} ;{ }^{1} \mathrm{H}$ NMR (CDCl, $\left.400 \mathrm{MHz}\right)$ $\delta$ 7.40-7.43 (m, 1H), 7.30-7.32 (m, 1H), 7.03-7.13 (m, 4H), 5.84-5.95 (m, 1H), 5.85 (s, 1H), 5.22 (dm, 1H, $J=17.2 \mathrm{~Hz}$ ), $5.17(\mathrm{dm}, 1 \mathrm{H}, J=10.2 \mathrm{~Hz}$ ), 4.51 (t, 2H, $J=7.8 \mathrm{~Hz}), 2.59$ (app qt, 
$2 \mathrm{H}, J=6.8,1.3 \mathrm{~Hz}$ ); ${ }^{13} \mathrm{C}$ NMR (APT, $\left.\mathrm{CDCl}_{3}, 100 \mathrm{MHz}\right) \delta 167.6,147.7,147.2,143.5,142.5$, 133.3, 125.6, 125.1, 120.5, 119.9, 117.8, 90.3, 82.4, 64.7, 33.0. HRMS (EI) calcd. for $\mathrm{C}_{15} \mathrm{H}_{14} \mathrm{O}_{3}\left(\mathrm{M}^{+}\right)$: 242.0943; found: 242.0942.

\section{Part C: Ruthenium-Catalyzed Reactions}

General procedure (B) for ruthenium-catalyzed formation of cyclobutene, cyclopropane and/or isochromene products. A mixture of alkene 2 (1.1-1.4 eq.), acetylene 4 (1.0 eq.) and $\mathrm{MeOH}$ (0.5 M versus acetylene) in an oven-dried vial was added via a cannula to an ovendried screw-cap vial containing $\mathrm{Cp} * \mathrm{Ru}(\mathrm{COD}) \mathrm{Cl}$ (weighed out from a dry box, 5-6 mol\%) under nitrogen. The reaction mixture was stirred at $60{ }^{\circ} \mathrm{C}$ for $1 \mathrm{~h}$. The solvent was evaporated and the crude product was purified by column chromatography.

General procedure $(C)$ for ruthenium-catalyzed formation of isochromene or cyclopropane products from ethyl diazoacetate. A solution of alkene 2 (1 eq.) in THF (0.5 M) in an ovendried vial was added via cannula to an oven-dried vial containing $\left[\mathrm{Cp} * \mathrm{Ru}\left(\mathrm{NCCH}_{3}\right)_{3}\right] \mathrm{PF}_{6}$ (weighed out from a dry box, 5-6 mol\%) under nitrogen. The reaction mixture was stirred at $60{ }^{\circ} \mathrm{C}$, then a solution of ethyl diazoacetate (2-3 eq.) in THF (1.1 M) was added over 30-45 min using a syringe. The reaction was monitored by ${ }^{1} \mathrm{H}$ NMR and upon completion, the solvent was evaporated and the crude product was purified by column chromatography.

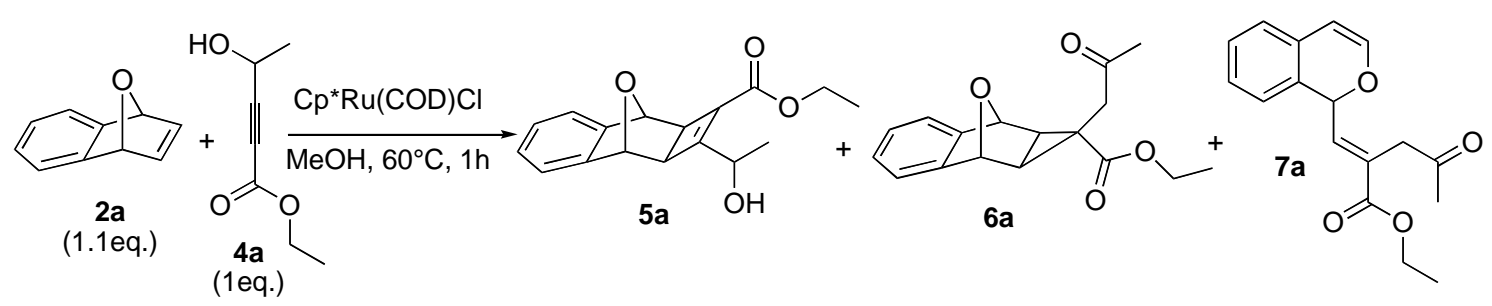

Cyclobutene 5a, cyclopropane 6a and isochromene 7a: Following the above general procedure (B) with alkene 2a (204.9 mg, $1.421 \mathrm{mmol})$, alkyne 4a (180.0 mg, $1.266 \mathrm{mmol}$ ), $\mathrm{MeOH}(1.6 \mathrm{~mL})$, and $\mathrm{Cp} * \mathrm{Ru}(\mathrm{COD}) \mathrm{Cl}(17.2 \mathrm{mg}, 0.0453 \mathrm{mmol})$. The reaction mixture was stirred at $60{ }^{\circ} \mathrm{C}$ for $1 \mathrm{~h}$. The crude product was purified by column chromatography (gradient EtOAc:hexanes $=1: 19$ to $1: 4)$ to provide $7 \mathbf{a}(181.2 \mathrm{mg}, 0.6330 \mathrm{mmol}, 50 \%)$ as a white solid (mp $=60-62{ }^{\circ} \mathrm{C}, \mathrm{Et}_{2} \mathrm{O} /$ Hexanes). 
5a (major diastereomer): $R_{f} 0.43$ (EtOAc:hexanes = 3:2); IR (neat) 3425, 3054, 2979, 2936, 1715, $1251 \mathrm{~cm}^{-1} ;{ }^{1} \mathrm{H}$ NMR ( $\left.\mathrm{CDCl}_{3}, 400 \mathrm{MHz}\right) \delta$ 7.28-7.34 (m, 2H), 7.17-7.22 (m, 2H), 5.11 (s, 1H), 5.02 (s, 1H), 4.72-4.83 (m, 2H), 4.29 (q, 2H, $J=7.1 \mathrm{~Hz}$ ), 2.81 (br dd, 1H, $J=3.4,1.0$ $\mathrm{Hz}$ ), 2.71 (br d, $1 \mathrm{H}, J=3.4 \mathrm{~Hz}$ ), 1.41 (d, 3H, $J=7.3 \mathrm{~Hz}$ ), 1.37 (t, 3H, $J=7.1 \mathrm{~Hz}$ ); ${ }^{13} \mathrm{C} \mathrm{NMR}$ (APT, $\left.\mathrm{CDCl}_{3}, 75 \mathrm{MHz}\right) \delta 166.7,163.3,144.1,143.8,129.5,126.9,126.8,119.9,119.7,75.9$, 75.3, 65.4, 61.1, 45.5, 44.4, 21.2, 14.2. Anal. calcd. for $\mathrm{C}_{17} \mathrm{H}_{18} \mathrm{O}_{4}=\mathrm{C}, 71.31 \%$; $\mathrm{H}, 6.34 \%$; found = C,71.10\%; H, 6.48\%. HRMS (CI) calcd. for $\mathrm{C}_{17} \mathrm{H}_{18} \mathrm{O}_{4}\left((\mathrm{M}+\mathrm{H})^{+}\right)$: 287.1283; found: 287.1280.

6a: $R_{f} 0.26$ (EtOAc:hexanes = 3:7); IR (neat) 3073, 3054, 2989, 2940, 1726, $1713 \mathrm{~cm}^{-1}$; ${ }^{1} \mathrm{H}$ NMR ( $\left.\mathrm{CDCl}_{3}, 400 \mathrm{MHz}\right) \delta 7.31$ (dd, 2H, $J=5.2,3.0 \mathrm{~Hz}$ ), 7.14 (dd, 2H, $J=5.2,3.0 \mathrm{~Hz}$ ), 5.23 (s, 2H), 4.03 (q, 2H, $J=7.1 \mathrm{~Hz}$ ), 3.42 (s, 2H), 2.20 (s, 3H), 1.98 (s, 2H), 1.16 (t, 3H, J = 7.1 $\mathrm{Hz}) ;{ }^{13} \mathrm{C}$ NMR (APT, $\left.\mathrm{CDCl}_{3}, 75 \mathrm{MHz}\right) \delta$ 207.1, 171.8, 147.3, 126.2, 119.7, 78.2, 61.2, 39.7, 39.4, 33.2, 30.1,14.0. HRMS (CI) calcd. for $\mathrm{C}_{17} \mathrm{H}_{18} \mathrm{O}_{4}\left((\mathrm{M}+\mathrm{H})^{+}\right)$: 287.1283; found: 287.1274. 7a: $R_{f} 0.33$ (EtOAc:hexanes=1:4); IR (neat) 3070, 2985, 2955, 2936, 1720, 1713, $1622 \mathrm{~cm}^{-1}$; ${ }^{1} \mathrm{H} \mathrm{NMR}\left(\mathrm{CDCl}_{3}, 400 \mathrm{MHz}\right) \delta$ 7.22-7.26 (m, 2H), 7.17 (app t, 1H, $J=7.5 \mathrm{~Hz}$ ), 6.99 (app t, 2H, $J=7.5 \mathrm{~Hz}$ ), 6.53 (d, 1H, $J=5.7 \mathrm{~Hz}), 5.84$ (d, 1H, $J=5.7 \mathrm{~Hz}), 5.73$ (d, 1H, $J=8.8 \mathrm{~Hz}$ ), 4.22 (q, 1H, $J=7.1 \mathrm{~Hz}), 4.21$ (q, 1H, $J=7.1 \mathrm{~Hz}$ ), 3.63 (d, 1H, $J=17.1 \mathrm{~Hz}), 3.53$ (d, 1H, $J=$ $17.1 \mathrm{~Hz}$ ), 2.23 (s, 3H), 1.29 (t, 3H, $J=7.1 \mathrm{~Hz}) ;{ }^{13} \mathrm{C}$ NMR (APT, $\left.\mathrm{CDCl}_{3}, 100 \mathrm{MHz}\right) \delta 204.5$, 166.5, 144.5, 139.5, 129.6, 129.4, 128.7, 128.0, 127.2, 124.4, 123.5, 105.5, 73.7, 61.4, 41.8, 29.9,14.1. HRMS (EI) calcd. for $\mathrm{C}_{17} \mathrm{H}_{18} \mathrm{O}_{4}\left(\mathrm{M}^{+}\right)$: 286.1205; found: 286.1207.
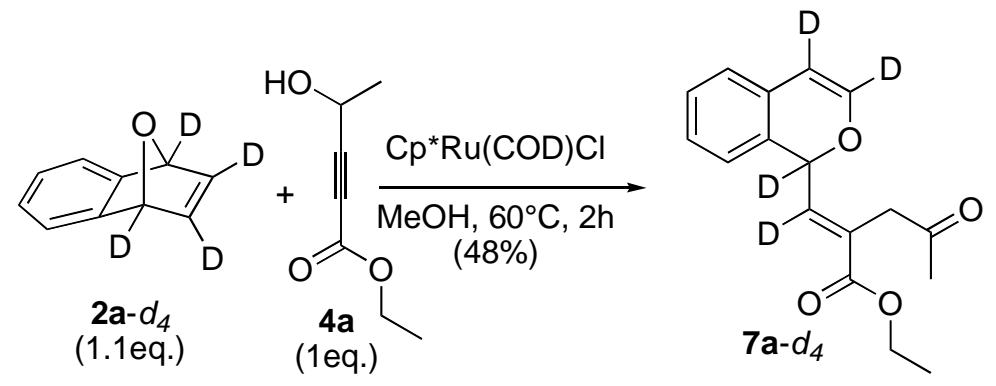

Isochromene 7a- $\boldsymbol{d}_{4}$ : Following the above general procedure (B) with alkene 2a- $d_{4}(59.0 \mathrm{mg}$, $0.399 \mathrm{mmol}$ ), alkyne 4a (50.5 mg, $0.355 \mathrm{mmol}), \mathrm{MeOH}(0.6 \mathrm{~mL})$, and $\mathrm{Cp} * \mathrm{Ru}(\mathrm{COD}) \mathrm{Cl}$ (9.6 $\mathrm{mg}, 0.025 \mathrm{mmol}$ ). The reaction mixture was stirred at $60{ }^{\circ} \mathrm{C}$ for $1 \mathrm{~h}$. The crude product was purified by column chromatography (gradient EtOAc:hexanes $=1: 9$ to $1: 4$ ) to provide $7 \mathbf{a}-d_{4}$ (49.5 mg, $0.170 \mathrm{mmol}$, 51\%). $\quad R_{f} 0.33$ (EtOAc:hexanes = 1:4); IR $\left(\mathrm{CH}_{2} \mathrm{Cl}_{2}\right)$ 3065, 2981, 2933, 1713, $1294 \mathrm{~cm}^{-1} ;{ }^{1} \mathrm{H}$ NMR $\left(\mathrm{CDCl}_{3}, 300 \mathrm{MHz}\right) \delta$ 7.14-7.26 (m, 2H), 6.96-7.01 (m, 2H), 
4.214 (q, 1H, $J=7.1 \mathrm{~Hz}), 4.209$ (q, 1H, $J=7.1 \mathrm{~Hz}), 3.63$ (d, 1H, $J=17.1 \mathrm{~Hz}), 3.52$ (d, 1H, $J$ $=17.1 \mathrm{~Hz}), 2.23(\mathrm{~s}, 3 \mathrm{H}), 1.28(\mathrm{t}, 3 \mathrm{H}, J=7.1 \mathrm{~Hz}) ;{ }^{13} \mathrm{C} \mathrm{NMR}\left(\mathrm{CDCl}_{3}, 75 \mathrm{MHz}\right) \delta 204.4,166.5$, $144.1(\mathrm{t}, J=29 \mathrm{~Hz}), 139.1(\mathrm{t}, J=25 \mathrm{~Hz}), 129.6,129.2,128.7,127.9,127.2,124.4,123.4$, $104.9(\mathrm{t}, J=25 \mathrm{~Hz}), 73.2(\mathrm{t}, J=22 \mathrm{~Hz}), 61.3,41.8,29.9,14.1 .{ }^{2} \mathrm{H} \mathrm{NMR}\left(\mathrm{CHCl}_{3}, 61 \mathrm{MHz}\right) \delta$ 6.57, 5.89, 5.74 (one deuterium signal is hidden under the $\mathrm{CDCl}_{3}$ peak). HRMS (EI) calcd. for $\mathrm{C}_{17} \mathrm{H}_{14}{ }^{2} \mathrm{H}_{4} \mathrm{O}_{4}\left(\mathrm{M}^{+}\right)$: 290.1454; found: 290.1457.
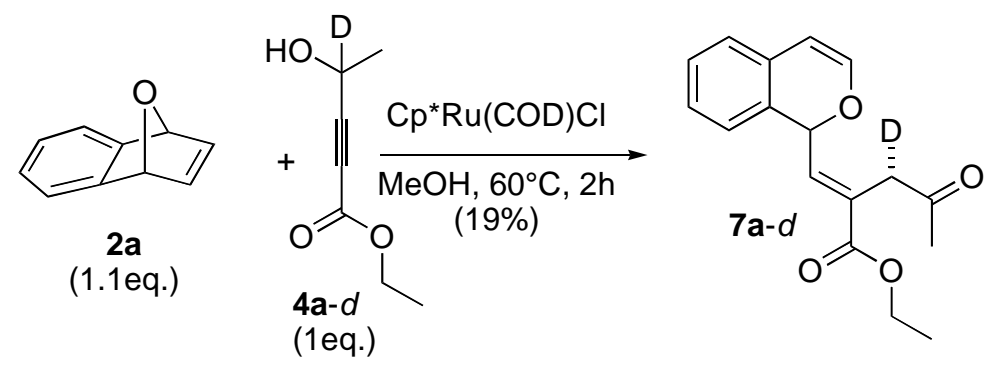

Isochromene 7a-d: Following the above general procedure (B) with alkene 2a (60.5 mg, $0.420 \mathrm{mmol}$ ), alkyne 4a-d (49.9 mg, $0.349 \mathrm{mmol}), \mathrm{MeOH}(0.6 \mathrm{~mL})$, and Cp*Ru(COD)Cl (6.3 $\mathrm{mg}, 0.017 \mathrm{mmol})$. The reaction mixture was stirred at $60{ }^{\circ} \mathrm{C}$ for $2.5 \mathrm{~h}$. The crude product was purified by column chromatography (gradient EtOAc:hexanes $=1: 9$ to $1: 4$ ) to provide $\mathbf{7 a}-d$ (19.5 mg, $0.0678 \mathrm{mmol}, 19 \%) .7 \mathrm{a}-d$ (major diastereomer) $R_{f} 0.33$ (EtOAc:hexanes=1:4); IR $\left(\mathrm{CH}_{2} \mathrm{Cl}_{2}\right)$ 3066, 2981, 2906, 1713, $1252 \mathrm{~cm}^{-1} ;{ }^{1} \mathrm{H}$ NMR $\left(\mathrm{CDCl}_{3}, 300 \mathrm{MHz}\right) \delta$ 7.13-7.25 (m, 3H), 6.97-7.01 (m, 2H), 6.53 (d, 1H, $J=5.7 \mathrm{~Hz}), 5.84$ (d, 1H, $J=5.7 \mathrm{~Hz}), 5.73$ (d, 1H, $J=8.8$ Hz), 4.222 (q, 1H, $J=7.1 \mathrm{~Hz}$ ), 4.218 (q, 1H, $J=7.1 \mathrm{~Hz}$ ), 3.61 (br s, 1H), 2.23 (s, 3H), 1.29 (t, $3 \mathrm{H}, J=7.1 \mathrm{~Hz}) ;{ }^{13} \mathrm{C} \mathrm{NMR}\left(\mathrm{CDCl}_{3}, 75 \mathrm{MHz}\right) \delta 204.5,166.5,144.5,139.5,129.7,129.4$, 128.7, 128.1, 127.2, 124.4, 123.5, 105.5, 73.7, 61.4, 41.6 (t, $J=20 \mathrm{~Hz}), 29.9,14.1 .{ }^{2} \mathrm{H} \mathrm{NMR}$ $\left(\mathrm{CHCl}_{3}, 61 \mathrm{MHz}\right) \delta$ 3.57. HRMS (EI) calcd. for $\mathrm{C}_{17} \mathrm{H}_{17}{ }^{2} \mathrm{HO}_{4}\left(\mathrm{M}^{+}\right)$: 287.1267; found: 287.1281.
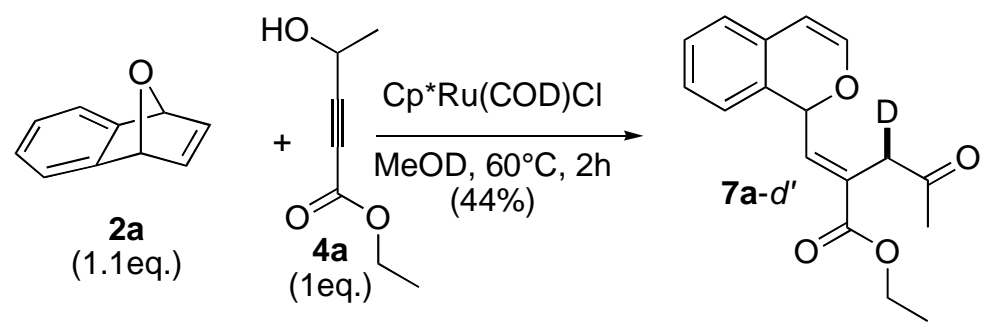

Isochromene 7a-d': Following the above general procedure (B) with alkene 2a (81.0 mg, $0.562 \mathrm{mmol}$ ), alkyne 4a (70.3 mg, $0.494 \mathrm{mmol})$, MeOD (0.7 mL), and Cp*Ru(COD)Cl (9.4 $\mathrm{mg}, 0.025 \mathrm{mmol})$. The reaction mixture was stirred at $60^{\circ} \mathrm{C}$ for $1 \mathrm{~h}$. The crude product was 
purified by column chromatography (gradient EtOAc:hexanes $=1: 9$ to $1: 4$ ) to provide $7 \mathbf{a}-d^{\prime}$ ' (62.3 mg, 0.217 mmol, 44\%). 7a-d' (major diastereomer) $R_{f} 0.33$ (EtOAc:hexanes = 1:4); IR $\left(\mathrm{CH}_{2} \mathrm{Cl}_{2}\right)$ 3066, 2981, 2906, 1713, $1552 \mathrm{~cm}^{-1}$; ${ }^{1} \mathrm{H} \mathrm{NMR}\left(\mathrm{CDCl}_{3}, 300 \mathrm{MHz}\right) \delta$ 7.12-7.25 (m, 3H), 6.97-7.07 (m, 2H), 6.53 (d, 1H, $J=5.7 \mathrm{~Hz}$ ), 5.84 (d, 1H, $J=5.7 \mathrm{~Hz}$ ), 5.74 (d, 1H, $J=8.8$ Hz), 4.215 (q, 1H, $J=7.1 \mathrm{~Hz}$ ), 4.210 (q, 1H, $J=7.1 \mathrm{~Hz}$ ), 3.52 (br s, 1H), 2.23 (s, 3H), 1.28 (t, $3 \mathrm{H}, J=7.1 \mathrm{~Hz}) ;{ }^{13} \mathrm{C} \mathrm{NMR}\left(\mathrm{CDCl}_{3}, 75 \mathrm{MHz}\right) \delta 204.5$, 166.5, 144.5, 139.5, 129.6, 129.3, 128.7, 128.0, 127.2, 124.4, 123.8, 105.4, 73.7, 61.3, 41.5 (t, $J=20 \mathrm{~Hz}), 29.9,14.1 .{ }^{2} \mathrm{H}$ NMR $\left(\mathrm{CHCl}_{3}, 61 \mathrm{MHz}\right) \delta$ 3.63. HRMS (EI) calcd. for $\mathrm{C}_{17} \mathrm{H}_{17}{ }^{2} \mathrm{HO}_{4}\left(\mathrm{M}^{+}\right)$: 287.1267; found: 287.1262.

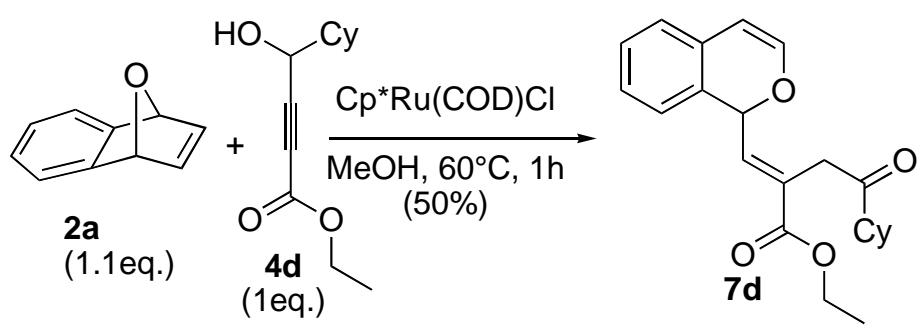

Isochromene 7d: Following the above general procedure (B) with alkene 2a (50.8 mg, 0.352 mmol), alkyne 4d (52.7 mg, $0.252 \mathrm{mmol}), \mathrm{MeOH}(0.7 \mathrm{~mL})$, and $\mathrm{Cp} * \mathrm{Ru}(\mathrm{COD}) \mathrm{Cl}$ (6.4 mg, $0.017 \mathrm{mmol})$. The reaction mixture was stirred at $60{ }^{\circ} \mathrm{C}$ for $1 \mathrm{~h}$. The crude product was purified by column chromatography (gradient $\mathrm{Et}_{2} \mathrm{O}$ :hexanes $=1: 9$ to $1: 4$ ) to provide $\mathbf{7 d}(44.2$ $\mathrm{mg}, 0.125 \mathrm{mmol}, 50 \%)$ as a white solid ( $\mathrm{mp}=60-62{ }^{\circ} \mathrm{C}$, uncrist.). $R_{f} 0.49$ (EtOAc:hexanes $=$ 3:7); IR $\left(\mathrm{CH}_{2} \mathrm{Cl}_{2}\right)$ 2981, 2929, 2853, $1709 \mathrm{~cm}^{-1} ;{ }^{1} \mathrm{H}$ NMR $\left(\mathrm{CDCl}_{3}, 400 \mathrm{MHz}\right) \delta$ 7.21-7.25 (m, 2H), 7.17 (app td, 1H, $J=7.6,1.3 \mathrm{~Hz}$ ), 6.99 (d, 2H, $J=7.5 \mathrm{~Hz}$ ), 6.53 (d, 1H, $J=5.7 \mathrm{~Hz}$ ), 5.84 (d, $1 \mathrm{H}, J=5.7 \mathrm{~Hz}$ ), 5.70 (d, 1H, $J=8.8 \mathrm{~Hz}), 4.20$ (q, 1H, $J=7.1 \mathrm{~Hz}$ ), 4.19 (q, 1H, $J=7.1$ Hz), 3.67 (d, 1H, $J=17.2 \mathrm{~Hz}$ ), 3.54 (d, 1H, $J=17.2 \mathrm{~Hz}$ ), 2.46 (tt, $1 \mathrm{H}, J=11.2,3.4 \mathrm{~Hz}$ ), 1.881.91 (m, 2H), 1.77-1.80 (m, 2H), 1.65-1.68 (m, 1H), 1.27 (t, 3H, $J=7.1 \mathrm{~Hz}$ ), 1.17-1.40 (m, $5 \mathrm{H}) ;{ }^{13} \mathrm{C} \mathrm{NMR}\left(\mathrm{APT}, \mathrm{CDCl}_{3}, 100 \mathrm{MHz}\right) \delta$ 209.5, 166.6, 144.6, 139.3, 129.8, 129.7, 128.6, 128.1, 127.2, 124.5, 123.4, 105.4, 73.8, 61.2, 50.8, 39.0, 28.5, 28.4, 25.8, 25.61, 25.55, 14.1. HRMS (CI) calcd. for $\mathrm{C}_{22} \mathrm{H}_{26} \mathrm{O}_{4}\left((\mathrm{M}+\mathrm{H})^{+}\right)$: 355.1909; found: 355.1915 . 

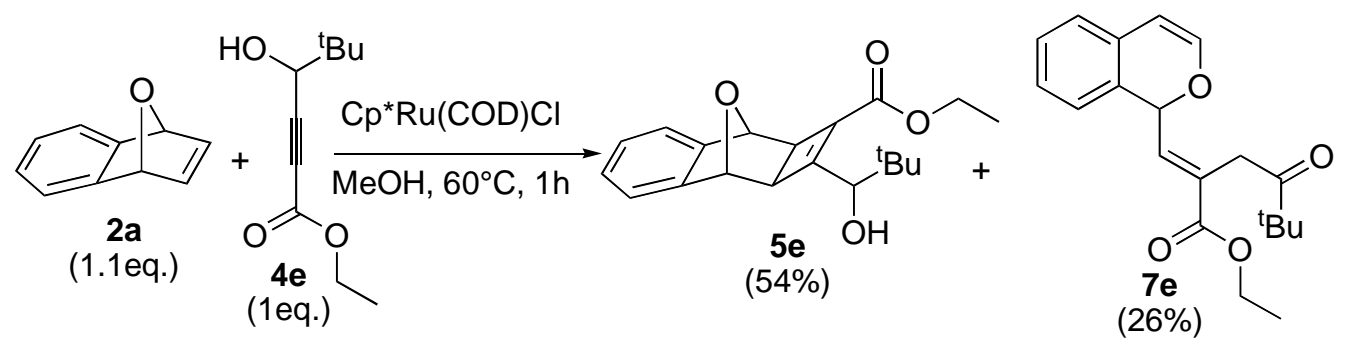

Cyclobutene 5e and isochromene 7e: Following the above general procedure (B) with alkene 2a (42.1 mg, $0.292 \mathrm{mmol}$ ), alkyne 4e (42.0 mg, $0.228 \mathrm{mmol})$, $\mathrm{MeOH}$ (0.6 mL), and $\mathrm{Cp} * \mathrm{Ru}(\mathrm{COD}) \mathrm{Cl}(6.2 \mathrm{mg}, 0.016 \mathrm{mmol})$. The reaction mixture was stirred at $60{ }^{\circ} \mathrm{C}$ for $1 \mathrm{~h}$. The crude product was purified by column chromatography (gradient $\mathrm{Et}_{2} \mathrm{O}$ :hexanes=1:9to2:3) to provide cycloadduct 5e (40.7 mg,0.124 mmol,54\%) and 7e (19.5 mg,0.0593 mmol,26\%).

5e (major diastereomer): white solid (mp $=88-89{ }^{\circ} \mathrm{C}$, uncrist.); $R_{f} 0.25$ (EtOAc:hexanes $=$ 2:3); IR $\left(\mathrm{CH}_{2} \mathrm{Cl}_{2}\right)$ 3408, 3058, 2963, 2906, 2869, 1712, $1682 \mathrm{~cm}^{-1} ;{ }^{1} \mathrm{H}$ NMR $\left(\mathrm{CDCl}_{3}, 400\right.$ MHz) $\delta$ 7.28-7.35 (m, 2H), 7.17-7.19 (m, 2H), 5.17 (d, 1H, J = 8.9 Hz), 5.09 (s, 1H), 5.08 (s, 1H), 4.27-4.31 (m, 2H), 4.01 (br d, 1H, $J=8.9 \mathrm{~Hz}$ ), 2.86 (br d, 1H, $J=3.2 \mathrm{~Hz}$ ), 2.70 (br d, $1 \mathrm{H}, J=3.4 \mathrm{~Hz}$ ), 1.37 (t, 3H, $J=7.1 \mathrm{~Hz}), 1.00(\mathrm{~s}, 9 \mathrm{H}) ;{ }^{13} \mathrm{C} \mathrm{NMR}\left(\mathrm{APT}, \mathrm{CDCl}_{3}, 100 \mathrm{MHz}\right) \delta$ 166.3, 163.5, 144.3, 143.9, 132.1, 126.9, 126.8, 120.0, 119.7, 78.4, 75.5, 75.2, 61.2, 49.0, 44.8, 37.4, 26.1, 14.2. HRMS (EI) calcd. for $\mathrm{C}_{20} \mathrm{H}_{24} \mathrm{O}_{4}\left(\mathrm{M}^{+}\right)$: 328.1675; found: 328.1670.

7e: colorless oil; $R_{f} 0.49$ (EtOAc:hexanes = 3:7); IR $\left(\mathrm{CH}_{2} \mathrm{Cl}_{2}\right)$ 3066, 2981, 2930, 2872, 1711 $\mathrm{cm}^{-1} ;{ }^{1} \mathrm{H} \mathrm{NMR}\left(\mathrm{CDCl}_{3}, 400 \mathrm{MHz}\right) \delta$ 7.21-7.25 (m, 2H), 7.18 (app td, 1H, $J=7.6,1.3 \mathrm{~Hz}$ ), 6.99 (d, 2H, $J=7.6 \mathrm{~Hz}$ ), 6.54 (d, 1H, $J=5.7 \mathrm{~Hz}$ ), 5.85 (d, 1H, $J=5.7 \mathrm{~Hz}$ ), 5.67 (d, 1H, $J=$ $8.8 \mathrm{~Hz}$ ), 4.20 (q, 1H, $J=7.1 \mathrm{~Hz}$ ), 4.19 (q, 1H, $J=7.1 \mathrm{~Hz}$ ), 3.75 (d, 1H, $J=17.6 \mathrm{~Hz}$ ), 3.59 (d, $1 \mathrm{H}, J=17.6 \mathrm{~Hz}$ ), 1.27 (t, 3H, $J=7.1 \mathrm{~Hz}), 1.20$ (s, 9H); ${ }^{13} \mathrm{C}$ NMR (APT, $\left.\mathrm{CDCl}_{3}, 100 \mathrm{MHz}\right) \delta$ 211.5, 166.6, 144.7, 139.2, 130.3, 129.7, 128.6, 128.2, 127.2, 124.5, 123.4, 105.4, 73.8, 61.2, 44.4, 35.4, 26.6, 14.1. HRMS (CI) calcd. for $\mathrm{C}_{20} \mathrm{H}_{24} \mathrm{O}_{4}\left((\mathrm{M}+\mathrm{H})^{+}\right)$: 329.1753; found: 329.1744.

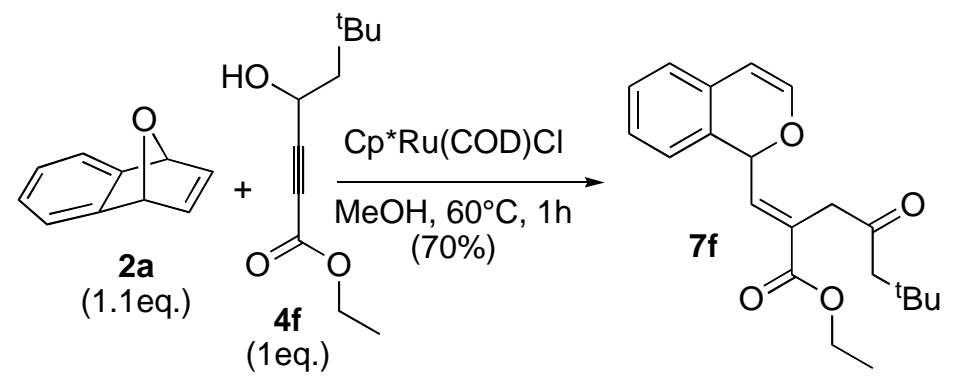

Isochromene 7f: Following the above general procedure (B) with alkene 2a (58.6 mg, 0.406 mmol), alkyne $4 f$ (55.8 mg, $0.281 \mathrm{mmol}), \mathrm{MeOH}(0.6 \mathrm{~mL})$, and Cp*Ru(COD)Cl (6.1 mg, 
$0.016 \mathrm{mmol}$ ). The reaction mixture was stirred at $60{ }^{\circ} \mathrm{C}$ for $1 \mathrm{~h}$. The crude product was purified by column chromatography (gradient $\mathrm{Et}_{2} \mathrm{O}$ :hexanes $=1: 9$ to 2:3) to provide $\mathbf{Z}$ (67.0 mg, 0.196 mmol, 70\%). 7f: white solid ( $\mathrm{mp}=52-55^{\circ} \mathrm{C}$, uncrist.); $R_{f} 0.45$ (EtOAc:hexanes = 1:9); IR $\left(\mathrm{CH}_{2} \mathrm{Cl}_{2}\right)$ 3065, 2954, 2869, 1713, $1626 \mathrm{~cm}^{-1} ;{ }^{1} \mathrm{H} \mathrm{NMR}\left(\mathrm{CDCl}_{3}, 400 \mathrm{MHz}\right) \delta 7.15-$ 7.25 (m, 3H), 6.98-7.00 (m, 2H), 6.53 (d, 1H, $J=5.7 \mathrm{~Hz}), 5.84$ (d, 1H, $J=5.7 \mathrm{~Hz}), 5.71$ (d, $1 \mathrm{H}, J=8.8 \mathrm{~Hz}$ ), 4.21 (q, 1H, $J=7.1 \mathrm{~Hz}), 4.20$ (q, 1H, $J=7.1 \mathrm{~Hz}$ ), 3.61 (d, $1 \mathrm{H}, J=17.3 \mathrm{~Hz}$ ), 3.50 (d, $1 \mathrm{H}, J=17.0 \mathrm{~Hz}$ ), 2.40 (s, 2H), 1.28 (t, 3H, $J=7.1 \mathrm{~Hz}), 1.03$ (s, 9H); ${ }^{13} \mathrm{C}$ NMR (APT, $\left.\mathrm{CDCl}_{3}, 100 \mathrm{MHz}\right) \delta$ 206.0, 166.6, 144.6, 139.2, 129.7, 129.6, 128.6, 128.1, 127.2, 124.5, 123.4, 105.4, 73.7, 61.2, 54.8, 43.2, 31.0, 29.6, 14.1. HRMS (CI) calcd. for $\mathrm{C}_{21} \mathrm{H}_{26} \mathrm{O}_{4}$ $\left((\mathrm{M}+\mathrm{H})^{+}\right)$: 343.1909; found: 343.1912 .

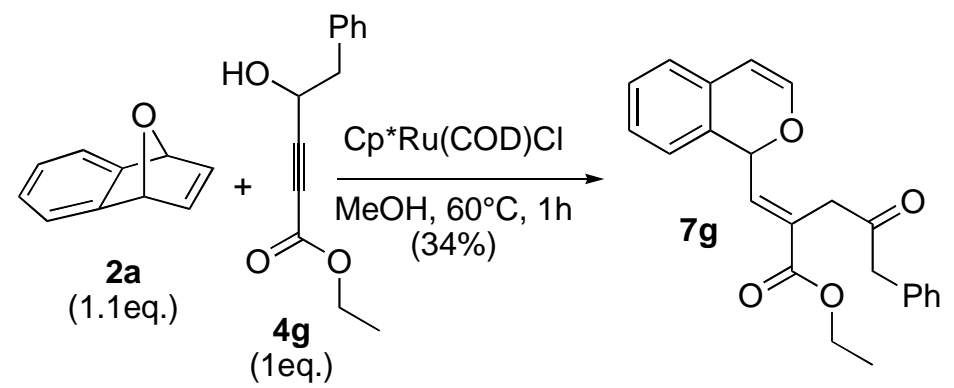

Isochromene 7g: Following the above general procedure (B) with alkene 2a (45.8 mg, 0.318 mmol), alkyne 4g (53.6 mg, $0.246 \mathrm{mmol}), \mathrm{MeOH}(0.7 \mathrm{~mL})$, and Cp*Ru(COD)Cl (6.3 mg, $0.017 \mathrm{mmol}$ ). The reaction mixture was stirred at $60{ }^{\circ} \mathrm{C}$ for $1 \mathrm{~h}$. The crude product was purified by column chromatography (gradient $\mathrm{Et}_{2} \mathrm{O}$ :hexanes $=1: 9$ to $2: 3$ ) to provide $\mathbf{7 g}$ (30.4 mg, $0.0839 \mathrm{mmol}$, 34\%) as a colorless oil. $R_{f} 0.34$ (EtOAc:hexanes = 1:4); IR $\left(\mathrm{CH}_{2} \mathrm{Cl}_{2}\right)$ 3064, 3028, 2980, 2936, 1722, $1714 \mathrm{~cm}^{-1}$; ${ }^{1} \mathrm{H}$ NMR $\left(\mathrm{CDCl}_{3}, 400 \mathrm{MHz}\right) \delta$ 7.13-7.26 (m, 7H), 7.07 (app td, 1H, $J=7.5,1.2 \mathrm{~Hz}$ ), 6.91 (d, 1H, $J=7.4 \mathrm{~Hz}$ ), 6.83 (d, 1H, $J=7.5 \mathrm{~Hz}), 6.41$ (d, 1H, $J$ $=5.7 \mathrm{~Hz}), 5.74(\mathrm{~d}, 1 \mathrm{H}, J=5.7 \mathrm{~Hz}), 5.53(\mathrm{~d}, 1 \mathrm{H}, J=8.8 \mathrm{~Hz}), 4.12$ (q, 1H, $J=7.1 \mathrm{~Hz}), 4.11(\mathrm{q}$, $1 \mathrm{H}, J=7.1 \mathrm{~Hz}$ ), 3.73 (s, 2H), 3.57 (d, 1H, $J=17.1 \mathrm{~Hz}$ ), 3.46 (d, 1H, $J=17.1 \mathrm{~Hz}$ ), 1.18 (t, 3H, $J=7.1 \mathrm{~Hz}) ;{ }^{13} \mathrm{C} \mathrm{NMR}\left(\mathrm{APT}, \mathrm{CDCl}_{3}, 100 \mathrm{MHz}\right) \delta 204.1$, 166.5, 144.5, 139.6, 133.8, 129.6, 129.5, 129.3, 128.74, 128.65, 128.0, 127.2, 127.1, 124.4, 123.4, 105.4, 73.6, 61.3, 50.0, 40.2, 14.1. HRMS (CI) calcd. for $\mathrm{C}_{23} \mathrm{H}_{22} \mathrm{O}_{4}\left((\mathrm{M}+\mathrm{H})^{+}\right)$: 363.1596; found: 363.1590 . 


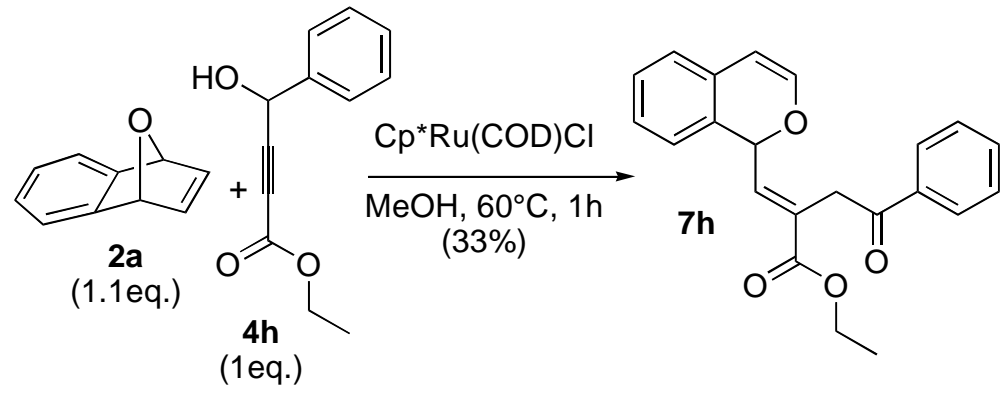

Isochromene 7h: Following the above general procedure (B) with alkene 2a (53.2 mg, 0.369 mmol), alkyne 4h (50.5 mg, $0.247 \mathrm{mmol}), \mathrm{MeOH}$ (0.5 mL), and Cp*Ru(COD)Cl (10.2 mg, $0.0268 \mathrm{mmol}$ ). The reaction mixture was stirred at $60{ }^{\circ} \mathrm{C}$ for $1 \mathrm{~h}$. The crude product was purified by column chromatography (gradient $\mathrm{Et}_{2} \mathrm{O}$ :hexanes $=1: 9$ to 2:3) to provide $7 \mathbf{h}$ (28.4 $\mathrm{mg}, 0.0815 \mathrm{mmol}, 33 \%)$ as a colorless oil. $R_{f} 0.29$ (EtOAc:hexanes = 1:4); $\mathrm{IR}\left(\mathrm{CH}_{2} \mathrm{Cl}_{2}\right)$ 3065, 2981, 2960, 1714, 1689, $1204 \mathrm{~cm}^{-1}$; ${ }^{1} \mathrm{H}$ NMR $\left(\mathrm{CDCl}_{3}, 400 \mathrm{MHz}\right) \delta 8.00$ (br d, 2H, $J=7.2$ Hz), 7.58 (app t, 1H, $J=7.3 \mathrm{~Hz}$ ), 7.45-7.50 (m, 2H), 7.32 (d, 2H, $J=8.8 \mathrm{~Hz}$ ), 7.16-7.24 (m, 3H), 7.05 (br d, 1H, $J=7.3 \mathrm{~Hz}$ ), 6.99 (br d, 1H, $J=7.2 \mathrm{~Hz}$ ), 6.54 (d, 1H, $J=5.7 \mathrm{~Hz}$ ), 5.84 (d, $1 \mathrm{H}, J=5.7 \mathrm{~Hz}), 5.78$ (d, 1H, $J=8.8 \mathrm{~Hz}), 4.26$ (d, 1H, $J=17.3 \mathrm{~Hz}), 4.17-4.25(\mathrm{~m}, 2 \mathrm{H}), 4.09$ (d, $1 \mathrm{H}, J=17.3 \mathrm{~Hz}), 1.25$ (t, 3H, $J=7.1 \mathrm{~Hz}$ ); ${ }^{13} \mathrm{C}$ NMR (APT, $\left.\mathrm{CDCl}_{3}, 100 \mathrm{MHz}\right) \delta 196.1$, 166.6, 144.6, 139.6, 136.5, 133.3, 129.9, 129.7, 128.6, 128.2, 128.1, 127.2, 124.5, 123.5, 105.5, 73.9, 61.3, 37.7, 14.1. HRMS (CI) calcd. for $\mathrm{C}_{22} \mathrm{H}_{20} \mathrm{O}_{4}\left(\mathrm{M}^{+}\right)$: 349.1440; found: 349.1446.

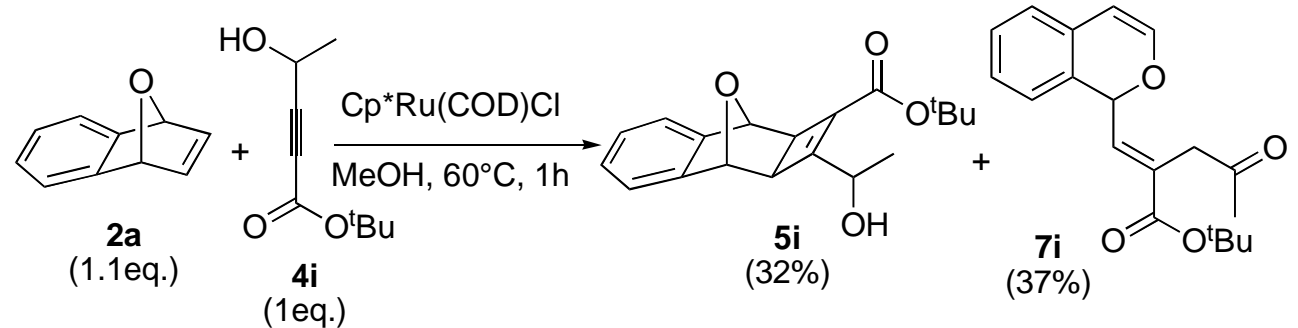

Cyclobutene 5i and isochromene 7i: Following the above general procedure (B) with alkene 2a (41.1 mg, $0.285 \mathrm{mmol}$ ), alkyne 4i (41.3 mg, $0.243 \mathrm{mmol})$, $\mathrm{MeOH}$ (0.6 mL), and $\mathrm{Cp} * \mathrm{Ru}(\mathrm{COD}) \mathrm{Cl}(5.8 \mathrm{mg}, 0.015 \mathrm{mmol})$. The reaction mixture was stirred at $60{ }^{\circ} \mathrm{C}$ for $1 \mathrm{~h}$. The crude product was purified by column chromatography (gradient $\mathrm{Et}_{2} \mathrm{O}$ :hexanes $=1: 9$ to 2:3) to provide cycloadduct $5 \mathbf{i}$ (24.4 mg, $0.0776 \mathrm{mmol}, 32 \%)$ and $7 \mathbf{i}(28.2 \mathrm{mg}, 0.0897 \mathrm{mmol}$, $37 \%)$.

$5 \mathbf{i}$ (major diastereomer): colorless oil; $R_{f} 0.18$ (EtOAc:hexanes = 1:4); IR $\left(\mathrm{CH}_{2} \mathrm{Cl}_{2}\right)$ 3415, 3058, 2976, 2931, $1712 \mathrm{~cm}^{-1}$; ${ }^{1} \mathrm{H}$ NMR $\left(\mathrm{CDCl}_{3}, 400 \mathrm{MHz}\right) \delta$ 7.27-7.32 (m, 2H), 7.17-7.20 (m, 
2H), 5.07 (s, 1H), 4.99 (s, 1H), 4.88 (br d, 1H, $J=3.2 \mathrm{~Hz}$ ), 4.73 (m, 1H), 2.75 (dd, 1H, $J=$ 3.4, $1.5 \mathrm{~Hz}$ ), 2.66 (br d, $1 \mathrm{H}, J=3.4 \mathrm{~Hz}$ ), 1.55 (s, 9H,), 1.40 (d, 3H, $J=6.9 \mathrm{~Hz}$ ); ${ }^{13} \mathrm{C} \mathrm{NMR}$ (APT, $\left.\mathrm{CDCl}_{3}, 100 \mathrm{MHz}\right) \delta 165.2,162.8,144.2,144.0,131.0,126.9,126.8,119.9,119.7,82.3$, 75.9, 75.2, 65.3, 45.0, 44.5, 28.2, 21.2. HRMS (EI) calcd. for $\mathrm{C}_{19} \mathrm{H}_{22} \mathrm{O}_{4}\left(\mathrm{M}^{+}\right)$: 314.1518; found: 314.1523 .

7i: white solid (mp $=97-98{ }^{\circ} \mathrm{C}$, uncrist.); $R_{f} 0.33$ (EtOAc:hexanes $=1: 9$ ); $\mathrm{IR}\left(\mathrm{CH}_{2} \mathrm{Cl}_{2}\right)$ 3065, 2984, 2936, 1713, 1621, $1160 \mathrm{~cm}^{-1}$; ${ }^{1} \mathrm{H}$ NMR $\left(\mathrm{CDCl}_{3}, 400 \mathrm{MHz}\right) \delta$ 7.14-7.25 (m, 3H), 6.977.01 (m, 2H), 6.54 (d, 1H, $J=5.7 \mathrm{~Hz}), 5.84$ (d, 1H, $J=5.7 \mathrm{~Hz}), 5.71$ (d, 1H, $J=8.8 \mathrm{~Hz}$ ), 3.57 (d, $1 \mathrm{H}, J=17.0 \mathrm{~Hz}$ ), 3.47 (d, $1 \mathrm{H}, J=17.0 \mathrm{~Hz}), 2.21$ (s, 3H), 1.48 (s, 9H); ${ }^{13} \mathrm{C}$ NMR (APT, $\left.\mathrm{CDCl}_{3}, 100 \mathrm{MHz}\right) \delta$ 204.6, 165.6, 144.6, 138.5, 131.0, 129.7, 128.6, 128.2, 127.2, 124.4, 123.5, 105.5, 81.6, 73.9, 42.0, 29.8, 28.0. HRMS (CI) calcd. for $\mathrm{C}_{19} \mathrm{H}_{22} \mathrm{O}_{4}\left((\mathrm{M}+\mathrm{H})^{+}\right)$: 315.1596; found: 315.1599 .



Cyclobutene $5 \mathbf{j}$, cyclopropane $\mathbf{6 j}$ and isochromene $7 \mathbf{j}$ : Following the above general procedure (B) with alkene $2 \mathbf{b}$ (112.4 $\mathrm{mg}, 0.550 \mathrm{mmol})$, alkyne 4a (70.5 mg, $0.496 \mathrm{mmol}$ ), $\mathrm{MeOH}(0.9 \mathrm{~mL})$, and $\mathrm{Cp} * \mathrm{Ru}(\mathrm{COD}) \mathrm{Cl}(13.3 \mathrm{mg}, 0.035 \mathrm{mmol})$. The reaction mixture was stirred at $60{ }^{\circ} \mathrm{C}$ for $1 \mathrm{~h}$. The crude product was purified by column chromatography (gradient $\mathrm{Et}_{2} \mathrm{O}$ :hexanes $=1: 9$ to $\left.1: 1\right)$ to provide $\mathbf{7 k}(103.1 \mathrm{mg}, 0.2976 \mathrm{mmol}, 60 \%)$.

$5 \mathbf{j}$ (major diastereomer): beige solid (mp $=120-122{ }^{\circ} \mathrm{C}$, uncrist.); $R_{f} 0.27$ (EtOAc:hexanes $=$ 3:2); IR $\left(\mathrm{CH}_{2} \mathrm{Cl}_{2}\right)$ 3413, 2975, 2906, 2836, 1712, 1501, $1260 \mathrm{~cm}^{-1} ;{ }^{1} \mathrm{H}$ NMR $\left(\mathrm{CDCl}_{3}, 400\right.$ MHz) $\delta 6.66$ (m, 2H), 5.27 (m, 1H), 5.17 (s, 1H), 4.89 (br d, 1H, J = 3.3 Hz), 4.75 (br q, 1H, $J$ $=6.9 \mathrm{~Hz}$ ), 4.28 (q, 2H, $J=7.2 \mathrm{~Hz}), 3.82$ (s, 3H), 3.79 (s, 3H), 2.84 (br dd, 1H, $J=3.4,1.4$ $\mathrm{Hz}$ ), 2.73 (br d, 1H, $J=3.2 \mathrm{~Hz}$ ), 1.41 (d, 3H, $J=6.9 \mathrm{~Hz}$ ), 1.36 (t, $3 \mathrm{H}, J=7.1 \mathrm{~Hz}$ ); ${ }^{13} \mathrm{C} \mathrm{NMR}$ (APT, $\left.\mathrm{CDCl}_{3}, 100 \mathrm{MHz}\right) \delta 167.1,163.5,147.4,147.2$, 133.2, 132.9, 129.7, 111.6, 111.3, 74.1, 73.4, 65.4, 61.1, 56.1, 55.9, 44.9, 43.9, 21.2, 14.3. HRMS (EI) calcd. for $\mathrm{C}_{19} \mathrm{H}_{22} \mathrm{O}_{6}\left(\mathrm{M}^{+}\right)$: 346.1416; found: 346.1423.

6j: white solid (mp $=84-87^{\circ} \mathrm{C}$, uncrist.); $R_{f} 0.23$ (EtOAc:hexanes=3:7); IR $\left(\mathrm{CH}_{2} \mathrm{Cl}_{2}\right)$ 2979, 2938, 2907, 1716, 1499, $1259 \mathrm{~cm}^{-1} ;{ }^{1} \mathrm{H} \mathrm{NMR}\left(\mathrm{CDCl}_{3}, 400 \mathrm{MHz}\right) \delta 6.65$ (s, 2H), 5.43 (s, 2H), 
4.03 (q, 2H, $J=7.1 \mathrm{~Hz}$ ), 3.78 (s, 6H), 3.38 (s, 2H), 2.18 (s, 3H), 2.02 (s, 2H), 1.16 (t, 3H, $J=$ $7.1 \mathrm{~Hz}) ;{ }^{13} \mathrm{C}$ NMR (APT, $\left.\mathrm{CDCl}_{3}, 75 \mathrm{MHz}\right) \delta$ 207.0, 171.9, 147.4, 136.4, 111.8, 76.5, 61.2, 56.3, 39.7, 39.3, 33.0, 30.1,14.1. Anal. calcd. for $\mathrm{C}_{19} \mathrm{H}_{22} \mathrm{O}_{6}=\mathrm{C}, 65.88 \%$; $\mathrm{H}, 6.40 \%$; found $=$ C,65.97\%; H, 6.21\%.

7j: colorless oil; $R_{f} 0.23$ (EtOAc:hexanes = 3:7); IR $\left(\mathrm{CH}_{2} \mathrm{Cl}_{2}\right)$ 3098, 3060, 2979, 2959, 2939, 2907, 2836, 1712, 1438, $1261 \mathrm{~cm}^{-1}$; ${ }^{1} \mathrm{H} \mathrm{NMR}\left(\mathrm{CDCl}_{3}, 400 \mathrm{MHz}\right) \delta 7.23$ (d, $\left.1 \mathrm{H}, J=9.4 \mathrm{~Hz}\right)$, 6.71 (d, 1H, $J=8.9 \mathrm{~Hz}), 6.62$ (d, 1H, $J=8.9 \mathrm{~Hz}), 6.45$ (d, 1H, $J=5.9 \mathrm{~Hz}), 6.09$ (d, 1H, $J=$ $9.4 \mathrm{~Hz}$ ), 6.08 (d, 1H, $J=5.8 \mathrm{~Hz}$ ), 4.12-4.18 (m, 2H), 3.78 (s, 3H), 3.70 (s, 3H), 3.63-3.74 (m, 2H), 2.21 (s, 3H), 1.24 (t, 3H, $J=7.1 \mathrm{~Hz}$ ); ${ }^{13} \mathrm{C}$ NMR (APT, $\left.\mathrm{CDCl}_{3}, 100 \mathrm{MHz}\right) \delta 204.9,167.1$, 148.7, 147.5, 143.2, 138.2, 127.0, 119.3, 116.7, 110.8, 109.1, 99.0, 68.1, 61.2, 56.0, 55.5, 41.8, 29.4,14.1. HRMS (CI) calcd. for $\mathrm{C}_{19} \mathrm{H}_{22} \mathrm{O}_{6}\left((\mathrm{M}+\mathrm{H})^{+}\right)$: 347.1495; found: 347.1486.

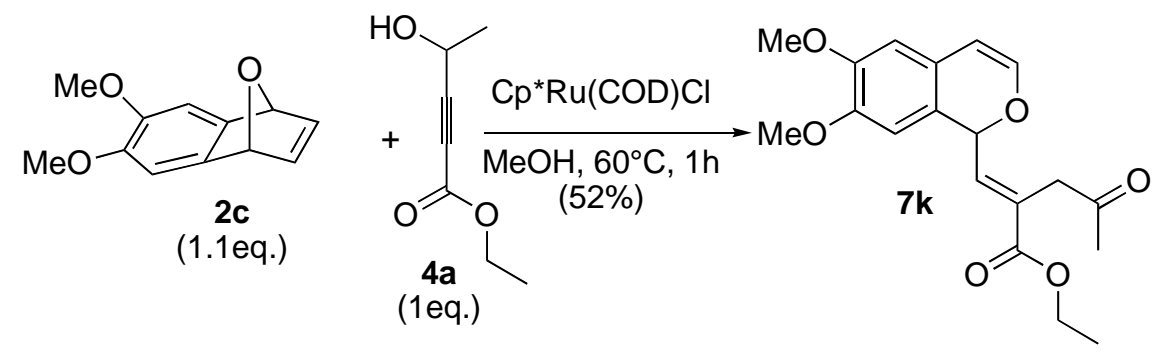

Isochromene 7k: Following the above general procedure (B) with alkene 2c (69.2 mg, 0.339 mmol), alkyne 4a (41.6 mg, $0.293 \mathrm{mmol}), \mathrm{MeOH}(1.2 \mathrm{~mL})$, and $\mathrm{Cp} * \mathrm{Ru}(\mathrm{COD}) \mathrm{Cl}$ (6.1 mg, $0.016 \mathrm{mmol}$ ). The reaction mixture was stirred at $60{ }^{\circ} \mathrm{C}$ for $1 \mathrm{~h}$. The crude product was purified by column chromatography (gradient EtOAc:hexanes $=1: 9$ to 2:3) to provide $7 \mathbf{l}$ (52.8 mg, $0.155 \mathrm{mmol}, 52 \%$ ) as a colorless oil. $R_{f} 0.50$ (EtOAc:hexanes $\left.=1: 1\right)$; IR $\left(\mathrm{CH}_{2} \mathrm{Cl}_{2}\right) 2984$, 2937, 2836, 1713, 1513, $1269 \mathrm{~cm}^{-1}$; ${ }^{1} \mathrm{H}$ NMR $\left(\mathrm{CDCl}_{3}, 400 \mathrm{MHz}\right) \delta 7.20$ (d, $\left.1 \mathrm{H}, J=8.8 \mathrm{~Hz}\right)$, 6.60 (s, 1H), 6.55 (s, 1H), 6.47 (d, 1H, $J=5.7 \mathrm{~Hz}), 5.76$ (d, 1H, $J=5.7 \mathrm{~Hz}), 5.69$ (d, 1H, $J=$ $8.8 \mathrm{~Hz}$ ), 4.18-4.24 (m, 2H), 3.861 (s, 3H), 3.858 (s, 3H), 3.70 (d, 1H, J = $17.0 \mathrm{~Hz}$ ), 3.45 (d, $1 \mathrm{H}, J=17.0 \mathrm{~Hz}), 2.23(\mathrm{~s}, 3 \mathrm{H}), 1.28(\mathrm{t}, 3 \mathrm{H}, J=7.1 \mathrm{~Hz}) ;{ }^{13} \mathrm{C} \mathrm{NMR}\left(\mathrm{APT}, \mathrm{CDCl}_{3}, 100 \mathrm{MHz}\right) \delta$ 204.7, 166.5, 149.0, 148.2, 142.8, 139.8, 128.9, 122.6, 120.0, 108.4, 107.2, 105.1, 73.5, 61.3, 56.2, 56.0, 41.8, 30.1,14.1. HRMS (CI) calcd. for $\mathrm{C}_{19} \mathrm{H}_{22} \mathrm{O}_{6}\left((\mathrm{M}+\mathrm{H})^{+}\right)$: 347.1495; found: 347.1485 . 


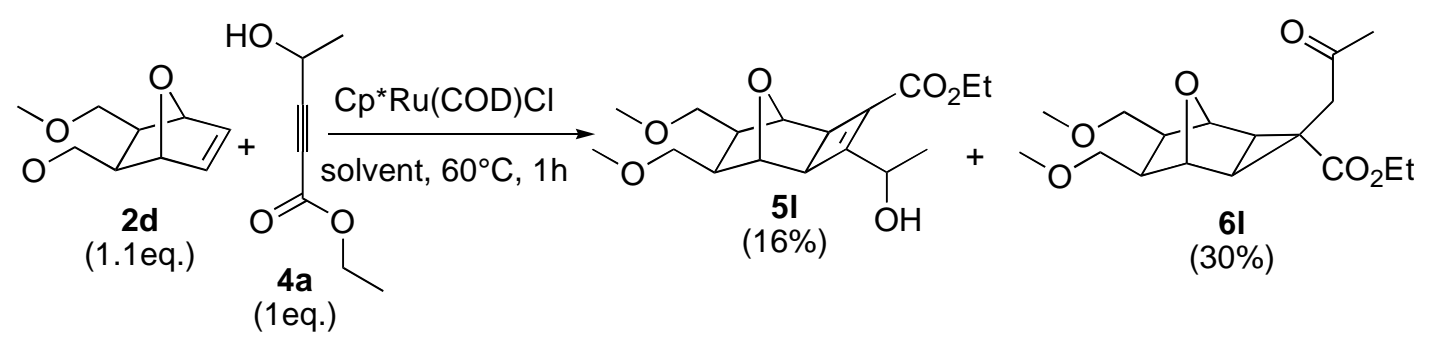

Cyclobutene 5l and cyclopropane 6l: Following the above general procedure (B) with alkene 2d (58.3 mg, $0.316 \mathrm{mmol}$ ), alkyne 4a (45.0 mg, $0.317 \mathrm{mmol})$, $\mathrm{MeOH}$ (0.8 mL), and $\mathrm{Cp} * \mathrm{Ru}(\mathrm{COD}) \mathrm{Cl}(6.1 \mathrm{mg}, 0.016 \mathrm{mmol})$. The reaction mixture was stirred at $60{ }^{\circ} \mathrm{C}$ for $1 \mathrm{~h}$. The crude product was purified by column chromatography (gradient $\mathrm{Et}_{2} \mathrm{O}$ :hexanes $=1: 9$ to 1:1) to provide cycloadduct $5 \mathbf{l}$ (16.1 mg, $0.0493 \mathrm{mmol}, 16 \%)$ and $6 \mathrm{~m}$ (31.0 mg, $0.0950 \mathrm{mmol}$, $30 \%)$.

$5 l$ (major diastereomer): pale yellow oil; $R_{f} 0.13$ (EtOAc:hexanes = 3:2); IR $\left(\mathrm{CH}_{2} \mathrm{Cl}_{2}\right)$ 3435, 2978, 2930, 2893, 2879, 2813, 1712, 1205, $1102 \mathrm{~cm}^{-1}$; ${ }^{1} \mathrm{H}$ NMR $\left(\mathrm{CDCl}_{3}, 400 \mathrm{MHz}\right) \delta 4.86$ (br d, 1H, $J=3.7 \mathrm{~Hz}), 4.54-4.56$ (m, 1H), 4.15-4.25 (m, 2H), 4.17 (s, 1H), 4.11 (s, 1H), 3.13-3.38 (m, 4H), 3.31 (s, 6H), 2.83-2.84 (m, 1H), 2.73 (br d, 1H, $J=3.3$ Hz), 1.93-2.14 (m, 2H), 1.30 (d, 3H, $J=6.8 \mathrm{~Hz}$ ), 1.28 (t, 3H, $J=7.1 \mathrm{~Hz}$ ); $\left.{ }^{13} \mathrm{C} \mathrm{NMR} \mathrm{(APT,} \mathrm{CDCl}_{3}, 100 \mathrm{MHz}\right) \delta 166.5$, 163.4, 128.4, 75.9, 75.3, 70.8, 70.5, 65.2, 60.9, 58.8, 47.3, 46.8, 45.6, 44.9, 44.5, 21.2, 14.2 . HRMS (EI) calcd. for $\mathrm{C}_{17} \mathrm{H}_{26} \mathrm{O}_{6}\left(\mathrm{M}^{+}\right)$: 326.1729; found: 326.1732 .

6l: pale yellow oil; $R_{f} 0.35$ (EtOAc:hexanes = 2:3); IR $\left(\mathrm{CH}_{2} \mathrm{Cl}_{2}\right)$ 2981, 2928, 2892, 2812, 1723, 1714, $1203 \mathrm{~cm}^{-1}$; ${ }^{1} \mathrm{H}$ NMR $\left(\mathrm{CDCl}_{3}, 400 \mathrm{MHz}\right) \delta 4.37$ (s, 2H), 4.04 (q, 2H, $J=7.1 \mathrm{~Hz}$ ), 3.28-3.32 (m, 2H), 3.29 (s, 6H), 3.18 (s, 2H), 3.16-3.21 (m, 2H), 2.25-2.27 (m, 2H), 2.11 (s, 3H), 1.81 (s, 2H), 1.17 (t, 3H, $J=7.1 \mathrm{~Hz}$ ); ${ }^{13} \mathrm{C}$ NMR (APT, $\mathrm{CDCl}_{3}, 100 \mathrm{MHz}$ ) $\delta$ 207.1, 173.1, 78.4, 70.4, 61.1, 58.7, 46.1, 38.9, 29.8, 29.7, 25.4,14.0. HRMS (EI) calcd. for $\mathrm{C}_{17} \mathrm{H}_{26} \mathrm{O}_{6}\left(\mathrm{M}^{+}\right)$: 326.1729; found: 326.1735 .

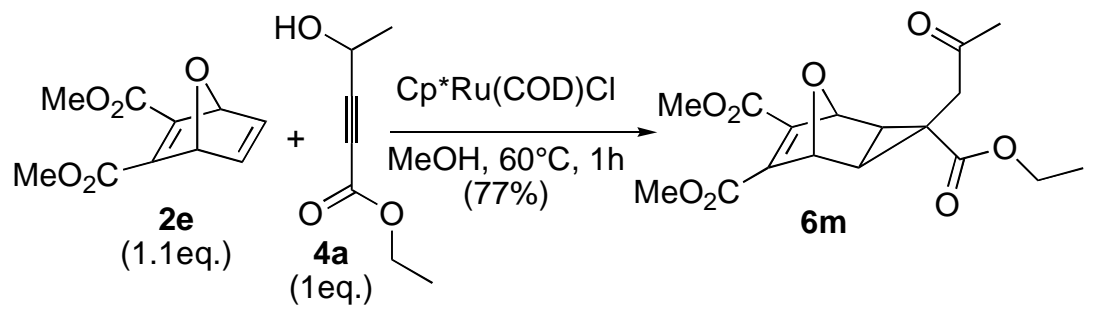

Cyclopropane 6m: Following the above general procedure (B) with alkene 2e (53.8 mg, $0.256 \mathrm{mmol}$ ), alkyne 4a (35.6 mg, $0.250 \mathrm{mmol}), \mathrm{MeOH}(0.6 \mathrm{~mL})$, and $\mathrm{Cp} * \mathrm{Ru}(\mathrm{COD}) \mathrm{Cl}$ (5.6 $\mathrm{mg}, 0.015 \mathrm{mmol}$ ). The reaction mixture was stirred at $60{ }^{\circ} \mathrm{C}$ for $1 \mathrm{~h}$. The crude product was 
purified by column chromatography (gradient EtOAc:hexanes $=1: 4$ to 2:3) to provide cypropane $\mathbf{6 m}$ (67.5 mg, $0.192 \mathrm{mmol}, \quad 77 \%)$ as a pale yellow oil. $R_{f} \quad 0.25$ (EtOAc:hexanes=2:3); IR $\left(\mathrm{CH}_{2} \mathrm{Cl}_{2}\right)$ 2984, 2954, 2847, 1721, 1715, $1628 \mathrm{~cm}^{-1}$; ${ }^{1} \mathrm{H}$ NMR $\left(\mathrm{CDCl}_{3}, 300 \mathrm{MHz}\right) \delta 5.15$ (s, 2H), 4.07 (q, 2H, J = $7.1 \mathrm{~Hz}$ ), 3.82 (s, 6H), 3.28 (s, 2H), 2.24 (s, 2H), 2.14 (s, 3H), 1.20 (t, 3H, $J=7.1 \mathrm{~Hz}) ;{ }^{13} \mathrm{C} \mathrm{NMR}\left(\mathrm{CDCl}_{3}, 75 \mathrm{MHz}\right) \delta 206.3,170.9,162.5$, 148.5, 80.7, 61.4, 52.3, 42.2, 39.4, 33.4, 30.0, 14.0. HRMS (CI) calcd. for $\mathrm{C}_{17} \mathrm{H}_{20} \mathrm{O}_{8}$ $\left((\mathrm{M}+\mathrm{H})^{+}\right)$: 353.1236; found: 353.1230.

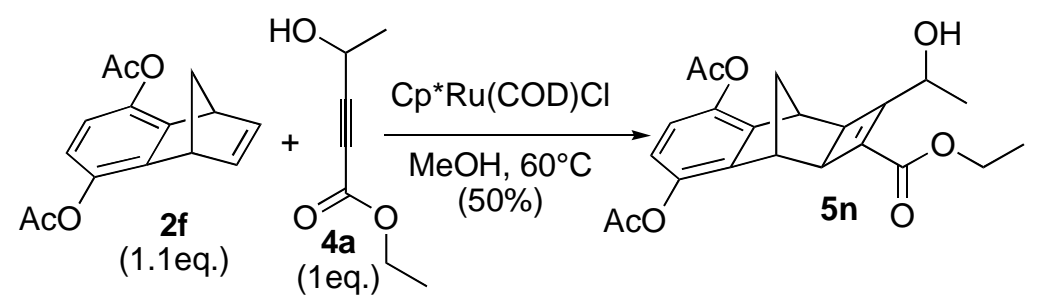

Cycloadduct 5n: Following the above general procedure (C) with alkene $2 \mathbf{f}$ (90.1 mg, 0.349 mmol), alkyne 2a (35.1 mg, $0.246 \mathrm{mmol})$, THF (0.6 mL), and Cp*Ru(COD)Cl (5.6 mg, 0.015 mmol). The reaction mixture was stirred at $60^{\circ} \mathrm{C}$ for $1 \mathrm{~h}$. The crude product was purified by column chromatography (gradient EtOAc:hexanes $=1: 9$ to 2:3) to provide cycloadduct 50 as an unseparable mixture of diastereomers (49.1 mg, $0.123 \mathrm{mmol}, 50 \%$ ). 5n (major diastereomer): $R_{f} 0.19$ (EtOAc:hexanes = 3:7); IR $\left(\mathrm{CH}_{2} \mathrm{Cl}_{2}\right) 3415$ (br), 2983, 2907, 1762, 1681, 1673, $1202 \mathrm{~cm}^{-1}$; ${ }^{1} \mathrm{H}$ NMR $\left(\mathrm{CDCl}_{3}, 300 \mathrm{MHz}\right) \delta 6.78$ (m, 2H), 4.79 (br s, 1H), 4.59 (br q, 1H, $J=6.7 \mathrm{~Hz}$ ), 4.23 (q, 2H, $J=7.1 \mathrm{~Hz}$ ), 3.18 (br s, 1H), 3.05 (br s, 1H), 2.76 (br d, 1H, $J$ = $3.1 \mathrm{~Hz}$ ), 2.70 (br d, 1H, J = 3.2 Hz), 2.31 (s, 3H), 2.30 (s, 3H), 1.77 (br s, 2H), 1.35 (d, 3H, $J=6.6 \mathrm{~Hz}), 1.32$ (t, 3H, $J=7.1 \mathrm{~Hz}) ;{ }^{13} \mathrm{C} \mathrm{NMR}\left(\mathrm{CDCl}_{3}, 75 \mathrm{MHz}\right) \delta 169.2,169.1,167.9,163.7$, 142.5, 142.3, 140.3, 139.8, 129.8, 120.5, 120.3, 65.7, 61.0, 44.2, 42.9, 40.6, 38.4, 37.9, 21.5, 20.84, 20.76, 14.1. HRMS (CI) calcd. for $\mathrm{C}_{22} \mathrm{H}_{24} \mathrm{O}_{7}\left((\mathrm{M}+\mathrm{H})^{+}\right)$: 401.1600; found: 401.1608.

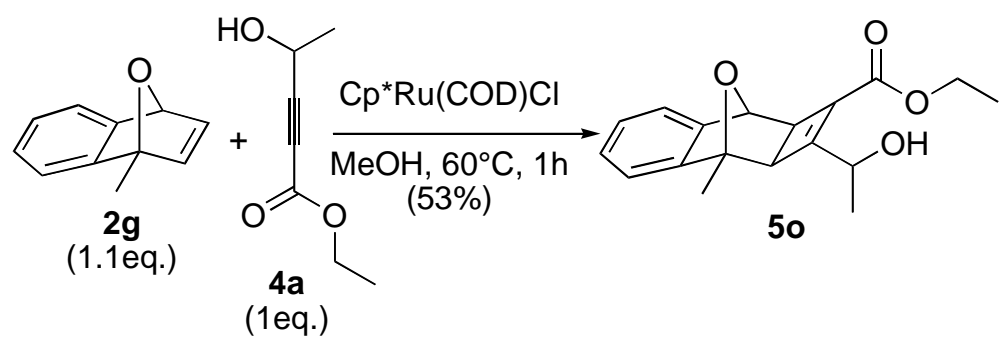

Cycloadduct 5o: Following the above general procedure (B) with alkene $\mathbf{2 g}$ (60.5 $\mathrm{mg}, 0.382$ mmol), alkyne 4a (41.8 mg, $0.294 \mathrm{mmol}), \mathrm{MeOH}(0.7 \mathrm{~mL})$, and $\mathrm{Cp} * \mathrm{Ru}(\mathrm{COD}) \mathrm{Cl}$ (5.7 mg, 
$0.015 \mathrm{mmol}$ ). The reaction mixture was stirred at $60{ }^{\circ} \mathrm{C}$ for $1 \mathrm{~h}$. The crude product was purified by column chromatography (gradient $\mathrm{Et}_{2} \mathrm{O}$ :hexanes $=1: 9$ to 2:3) to provide 50 (46.8 mg, $0.156 \mathrm{mmol}, 53 \%)$ as a pale yellow oil. $R_{f} 0.13$ (EtOAc:hexanes $\left.=1: 9\right)$; IR $\left(\mathrm{CH}_{2} \mathrm{Cl}_{2}\right)$ 3412, 3058, 2981, 2929, 1713, $1674 \mathrm{~cm}^{-1}$; ${ }^{1} \mathrm{H}$ NMR $\left(\mathrm{CDCl}_{3}, 400 \mathrm{MHz}\right) \delta$ 7.30-7.33 (m, 1H), 7.20-7.22 (m, 3H), 5.48 (br d, 1H, $J=2.8 \mathrm{~Hz}$ ), 5.05 (s, 1H), 4.74 (m, $1 \mathrm{H}$ ), 4.30 (q, 1H, $J=$ 7.1Hz), 4.29 (q, 1H, $J=7.1 \mathrm{~Hz}$ ), 2.84 (dd, 1H, $J=3.3,1.6 \mathrm{~Hz}$ ), 2.60 (br d, $1 \mathrm{H}, J=3.3 \mathrm{~Hz}$ ), 1.75 (s, 3H), 1.38 (d, 3H, $J=6.9 \mathrm{~Hz}$ ), 1.37 (t, 3H, $J=7.1 \mathrm{~Hz}$ ); ${ }^{13} \mathrm{C}$ NMR (APT, $\mathrm{CDCl}_{3}, 100$ MHz) $\delta 167.4,163.7,146.7,144.9,129.9,126.9,119.8,118.5,82.1,75.7,66.7,61.2,48.8$, 47.0, 21.0, 15.4, 14.2. HRMS (EI) calcd. for $\mathrm{C}_{18} \mathrm{H}_{20} \mathrm{O}_{4}\left(\mathrm{M}^{+}\right)$: 300.1362; found: 300é1368.
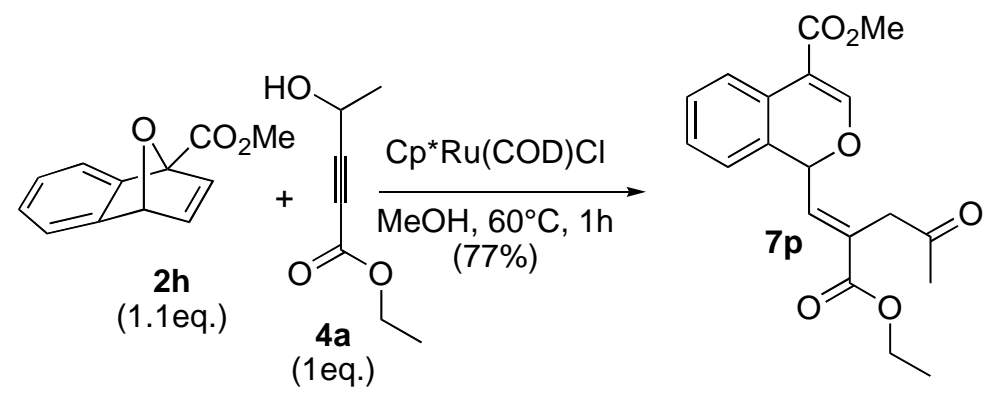

Isochromene 7p: Following the above general procedure (B) with alkene $\mathbf{2 h}$ (55.9 $\mathrm{mg}, 0.276$ mmol), alkyne 4a (38.6 mg, $0.272 \mathrm{mmol}), \mathrm{MeOH}(0.6 \mathrm{~mL})$, and $\mathrm{Cp} * \mathrm{Ru}(\mathrm{COD}) \mathrm{Cl}$ (5.8 mg, $0.015 \mathrm{mmol})$. The reaction mixture was stirred at $60{ }^{\circ} \mathrm{C}$ for $1 \mathrm{~h}$. The crude product was purified by column chromatography (gradient $\mathrm{Et}_{2} \mathrm{O}$ :hexanes $=1: 4$ to $1: 1$ ) to provide $\mathbf{7 p}(72.1$ mg, $0.209 \mathrm{mmol}, 77 \%)$ as a white solid (mp $=100-101{ }^{\circ} \mathrm{C}_{2} \mathrm{Et}_{2} \mathrm{O} /$ hexanes). $R_{f} 0.24$ (EtOAc:hexanes = 1:4); IR $\left(\mathrm{CH}_{2} \mathrm{Cl}_{2}\right)$ 3064, 2982, 2952, 1721, 1717, $1210 \mathrm{~cm}^{-1} ;{ }^{1} \mathrm{H}$ NMR $\left(\mathrm{CDCl}_{3}, 400 \mathrm{MHz}\right) \delta$ 7.26-7.32 (m, 2H), 7.15-7.18 (m, 2H), 7.08-7.10 (m, 1H), 6.97 (s, 1H), 5.87 (d, 1H, $J=8.6 \mathrm{~Hz}), 4.19$ (q, 1H, $J=7.1 \mathrm{~Hz}), 4.18$ (q, 1H, $J=7.1 \mathrm{~Hz}$ ), 3.83 (s, 3H), 3.71 (d, $1 \mathrm{H}, J=17.1 \mathrm{~Hz}$ ), 3.52 (d, $1 \mathrm{H}, J=17.1 \mathrm{~Hz}$ ), 2.23 (s, 3H), 1.26 (t, 3H, $J=7.1 \mathrm{~Hz}) ;{ }^{13} \mathrm{C}$ NMR (APT, $\left.\mathrm{CDCl}_{3}, 100 \mathrm{MHz}\right) \delta$ 204.5, 166.2, 162.9, 142.5, 138.4, 130.1, 129.7, 129.4, 128.9, 128.7, 125.8, 124.7, 113.0, 74.8, 61.3, 52.3, 41.8, 30.0,14.0. HRMS (CI) calcd. for $\mathrm{C}_{19} \mathrm{H}_{20} \mathrm{O}_{6}\left((\mathrm{M}+\mathrm{H})^{+}\right)$: 345.1338; found: 345.1326. Anal. calcd. for $\mathrm{C}_{19} \mathrm{H}_{20} \mathrm{O}_{6}=\mathrm{C}, 66.27 \%$; $\mathrm{H}$, 5.85\%; found = C,66.40\%; H, 5.72\%. 

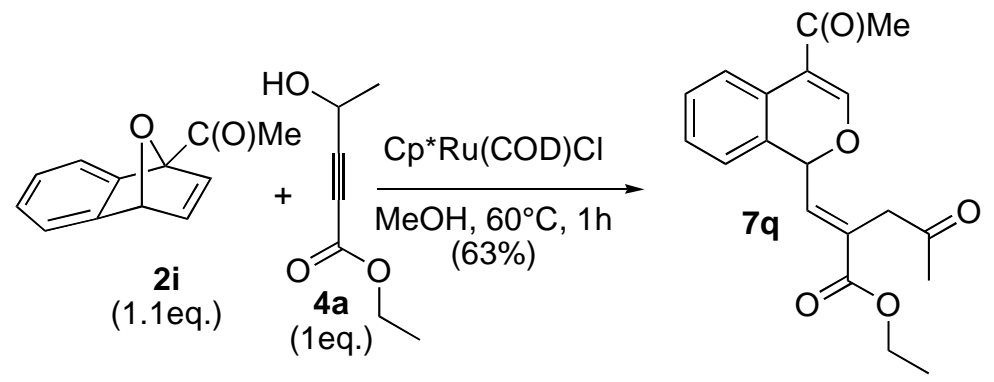

Isochromene 7q: Following the above general procedure (B) with alkene $2 \mathbf{2 i}$ (73.4 mg, 0.394 mmol), alkyne 4a (42.3 mg, $0.298 \mathrm{mmol}), \mathrm{MeOH}(0.8 \mathrm{~mL}$ ), and $\mathrm{Cp} * \mathrm{Ru}(\mathrm{COD}) \mathrm{Cl}$ (6.7 mg, $0.018 \mathrm{mmol}$ ). The reaction mixture was stirred at $60{ }^{\circ} \mathrm{C}$ for $1 \mathrm{~h}$. The crude product was purified by column chromatography (gradient EtOAc:hexanes $=1: 9$ to $2: 3$ ) to provide $\mathbf{7 q}$ (61.6 mg, $0.188 \mathrm{mmol}, 63 \%$ ) as a white solid (m.p. $=99-102{ }^{\circ} \mathrm{C}, \mathrm{Et}_{2} \mathrm{O} /$ hexanes). $\quad R_{f} 0.30$ (EtOAc:hexanes = 3:7); IR $\left(\mathrm{CH}_{2} \mathrm{Cl}_{2}\right)$ 3079, 2981, 2933, 1714, 1681, $1256 \mathrm{~cm}^{-1} ;{ }^{1} \mathrm{H}$ NMR $\left(\mathrm{CDCl}_{3}, 400 \mathrm{MHz}\right) \delta$ 7.27-7.31 (m, 2H), 7.19-7.21 (m, 1H), 7.16 (d, $\left.1 \mathrm{H}, J=8.8 \mathrm{~Hz}\right), 7.07-$ 7.09 (m, 1H), 6.85 (s, 1H), 5.84 (d, 1H, $J=8.8 \mathrm{~Hz}), 4.203$ (q, 1H, $J=7.1 \mathrm{~Hz}), 4.199$ (q, 1H, $J$ = $7.1 \mathrm{~Hz}$ ), 3.68 (d, $1 \mathrm{H}, J=17.1 \mathrm{~Hz}), 3.53(\mathrm{~d}, 1 \mathrm{H}, J=17.1 \mathrm{~Hz}), 2.34$ (s, 3H), $2.24(\mathrm{~s}, 3 \mathrm{H}), 1.27$ (t, 3H, $J=7.1 \mathrm{~Hz}$ ); ${ }^{13} \mathrm{C}$ NMR (APT, $\left.\mathrm{CDCl}_{3}, 100 \mathrm{MHz}\right) \delta 204.4,193.9,166.3,149.0,138.6$, 130.0, 129.9, 129.6, 129.0, 128.8, 126.4, 124.6, 111.0, 74.5, 61.4, 41.8, 30.0, 25.7, 14.0. Anal. calcd. for $\mathrm{C}_{19} \mathrm{H}_{20} \mathrm{O}_{5}=\mathrm{C}, 69.50 \% ; \mathrm{H}, 6.14 \%$; found = C,69.67\%; $\mathrm{H}, 6.01 \%$.
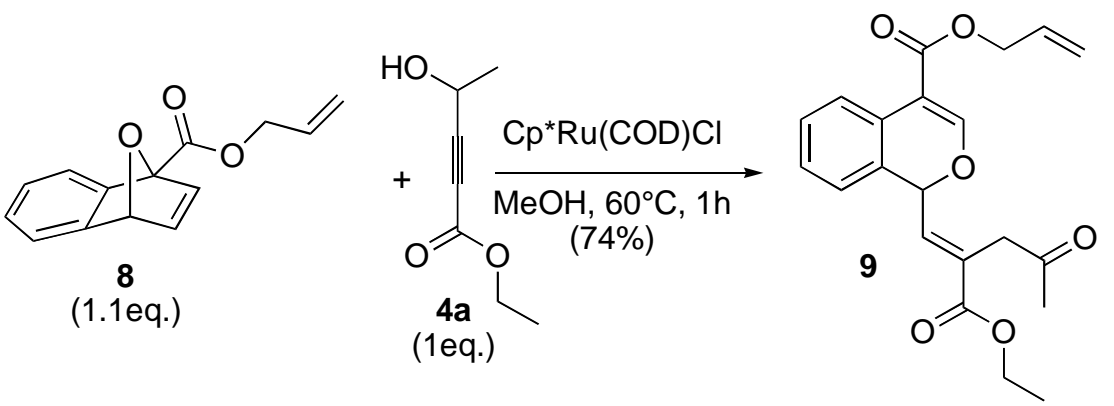

Isochromene 9: Following the above general procedure (B) with alkene 8 (50.9 mg, 0.210 mmol), alkyne 4a (27.1 mg, $0.191 \mathrm{mmol}), \mathrm{MeOH}(0.5 \mathrm{~mL})$, and $\mathrm{Cp} * \mathrm{Ru}(\mathrm{COD}) \mathrm{Cl}$ (5.2 mg, $0.0137 \mathrm{mmol}$ ). The reaction mixture was stirred at $60^{\circ} \mathrm{C}$ for $1 \mathrm{~h}$. The crude product was purified by column chromatography (gradient $\mathrm{Et}_{2} \mathrm{O}$ :hexanes $=1: 9$ to 2:3) to provide 9 (51.9 mg, $0.141 \mathrm{mmol}, 74 \%$ ) as a white solid ( $\mathrm{mp}=55-57{ }^{\circ} \mathrm{C}, \mathrm{Et}_{2} \mathrm{O} /$ hexanes). $R_{f} 0.39$ (EtOAc:hexanes = 1:4); IR $\left(\mathrm{CH}_{2} \mathrm{Cl}_{2}\right)$ 3076, 2981, 2904, 1722, $1714 \mathrm{~cm}^{-1} ;{ }^{1} \mathrm{H}$ NMR $\left(\mathrm{CDCl}_{3}\right.$, $400 \mathrm{MHz}) \delta$ 7.30-7.32 (m, 2H), 7.16-7.19 (m, 2H), 7.09-7.11 (m, 1H), 6.95 (s, 1H), 5.88 (d, $1 \mathrm{H}, J=8.6 \mathrm{~Hz}$ ), 5.79-5.87 (m, 1H), 5.16 (dd, $1 \mathrm{H}, J=17.1,1.6 \mathrm{~Hz}$ ), 5.11 (dd, $1 \mathrm{H}, J=10.3$, 
$1.5 \mathrm{~Hz}), 4.81$ (t, 2H, $J=6.8 \mathrm{~Hz}), 4.17-4.24$ (m, 2H), 3.73 (d, 1H, $J=17.1 \mathrm{~Hz}), 3.54$ (d, 1H, $J$ $=17.1 \mathrm{~Hz}$ ), 2.49 (app qt, $2 \mathrm{H}, J=6.8,1.2 \mathrm{~Hz}), 2.25$ (s, 3H), $1.28(\mathrm{t}, 3 \mathrm{H}, J=7.1 \mathrm{~Hz}) ;{ }^{13} \mathrm{C}$ NMR $\left(\mathrm{APT}, \mathrm{CDCl}_{3}, 100 \mathrm{MHz}\right) \delta 204.6,166.3,162.4,142.6,138.6,133.6,130.0,129.7,129.5$, 128.9, 128.7, 125.8, 124.7, 117.5, 112.9, 74.8, 64.4, 61.4, 41.9, 33.0, 30.0, 14.1. HRMS (CI) calcd. for $\mathrm{C}_{22} \mathrm{H}_{24} \mathrm{O}_{6}\left((\mathrm{M}+\mathrm{H})^{+}\right)$: 385.1651; found: 385.1665 .

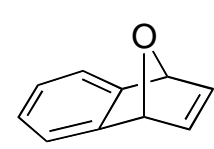

$2 a$

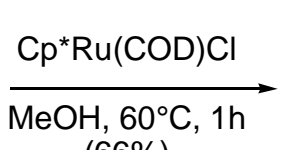

$(66 \%)$<smiles>CO[C@H]1C=Cc2ccccc2C1O</smiles>

10

(racemic)

Ruthenium-catalyzed nucleophilic addition of methanol on 2a: a solution of alkene 2a (36.5 mg, $0.253 \mathrm{mmol}$ ) and methanol $(0.6 \mathrm{~mL})$ in an oven-dried vial was added via a cannula to an oven-dried vial containing $\mathrm{Cp} * \mathrm{Ru}(\mathrm{COD}) \mathrm{Cl}$ (weighed out from a dry box, $5.9 \mathrm{mg}, 0.016$ mmol) under nitrogen. The reaction mixture was heated at $60^{\circ} \mathrm{C}$ for $1 \mathrm{~h}$. The crude product was purified by column chromatography (EtOAc:hexanes = 1:9) to give 10 (29.3 mg, 0.166 mmol, 66\%) as a white solid ( $\mathrm{mp}=48-50{ }^{\circ} \mathrm{C}$, uncrist.). $R_{f} 0.21$ (EtOAc:hexanes = 1:4); IR $\left(\mathrm{CH}_{2} \mathrm{Cl}_{2}\right)$ 3420, 3037, 2979, 2929, 2825, $1455 \mathrm{~cm}^{-1} ;{ }^{1} \mathrm{H}$ NMR $\left(\mathrm{CDCl}_{3}, 400 \mathrm{MHz}\right) \delta 7.58$ (d, $1 \mathrm{H}, J=6.8 \mathrm{~Hz}$ ), 7.24-7.27 (m, 2H), 7.08-7.10 (m, 1H), 6.48 (dd, 1H, $J=9.9,2.0 \mathrm{~Hz}$ ), 6.06 (dd, 1H, $J=9.9,2.3 \mathrm{~Hz}$ ), 4.91 (d, 1H, $J=10.2 \mathrm{~Hz}$ ), 4.11 (app dt, 1H, $J=10.3,2.2 \mathrm{~Hz}$ ), 3.51 (s, 3H), 2.64 (br s, $1 \mathrm{H}$ ); ${ }^{13} \mathrm{C}$ NMR (APT, $\left.\mathrm{CDCl}_{3}, 100 \mathrm{MHz}\right) \delta 135.8,131.9,128.3,127.9$, 127.8, 126.7, 126.3, 125.0, 82.2, 72.4, 56.8. HRMS (EI) calcd. for $\mathrm{C}_{11} \mathrm{H}_{12} \mathrm{O}_{2}\left(\mathrm{M}^{+}\right)$176.0837; found: 176.0840 . Spectral data are in agreement with the literature values. ${ }^{11}$

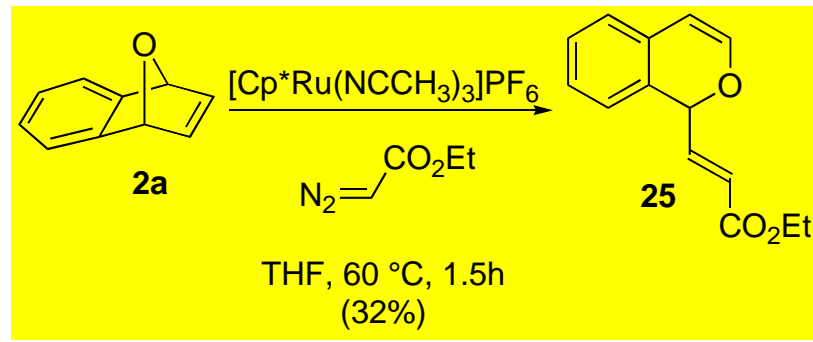

Isochromene 25: Following the above general procedure (C) with alkene 2a (151.3 mg, 1.049 mmol), THF (1.0 mL), [Cp*Ru( $\left.\left.\mathrm{NCCH}_{3}\right)_{3}\right] \mathrm{PF}_{6}$ (30.2 mg, $\left.0.0599 \mathrm{mmol}\right)$, ethyl diazoacetate (250 $\mu \mathrm{L}, 2.41 \mathrm{mmol})$ and THF $(2.1 \mathrm{~mL})$. The addition was done over $45 \mathrm{~min}$ and the reaction mixture was stirred at $60{ }^{\circ} \mathrm{C}$ for $1.5 \mathrm{~h}$. The crude product was purified by column

\footnotetext{
${ }^{11}$ Kende, A.; S.; Belletire, J.; Bentley, J.; Hume, E.; Airey, J. J. Am. Chem. Soc. 1975, 97, 4427.
} 
chromatography (gradient $\mathrm{Et}_{2} \mathrm{O}$ :hexanes = 1:19 to 1:9) to provide 25 (76.7 $\mathrm{mg}, 0.333 \mathrm{mmol}$, 32\%) as a colorless oil. If diethyl fumarate and/or diethyl maleate are still present at this stage, they were distilled off under reduced pressure (kugelrohr distillation). $R_{f} 0.45$ (EtOAc:hexanes = 1:9); IR (neat) 3070, 2981, 2937, 2903, 1720, 1628, $1274 \mathrm{~cm}^{-1} ;{ }^{1} \mathrm{H}$ NMR $\left(\mathrm{CDCl}_{3}, 400 \mathrm{MHz}\right) \delta 7.16-7.27(\mathrm{~m}, 2 \mathrm{H}), 7.06(\mathrm{dd}, 1 \mathrm{H}, J=1.5,5.2 \mathrm{~Hz}), 6.97-7.01(\mathrm{~m}, 2 \mathrm{H})$, $6.54(\mathrm{~d}, 1 \mathrm{H}, J=5.7 \mathrm{~Hz}), 5.96$ (d, 1H, $J=15.7 \mathrm{~Hz}), 5.78$ (d, 1H, $J=5.7 \mathrm{~Hz}), 5.72$ (d, 1H, $J=$ $5.0 \mathrm{~Hz}), 4.19$ (q, 2H, $J=7.1 \mathrm{~Hz}), 1.28$ (t, 3H, $J=7.1 \mathrm{~Hz}) ;{ }^{13} \mathrm{C}$ NMR (APT, $\left.\mathrm{CDCl}_{3}, 100 \mathrm{MHz}\right)$ $\delta$ 166.0, 144.2, 144.0, 129.3, 128.7, 127.9, 127.1, 124.7, 123.7, 122.3, 104.9, 75.9, 60.6, 14.2 . HRMS (CI) calcd. for $\mathrm{C}_{14} \mathrm{H}_{14} \mathrm{O}_{3}\left((\mathrm{M}+\mathrm{H})^{+}\right)$: 231.1021; found: 231.1025 .

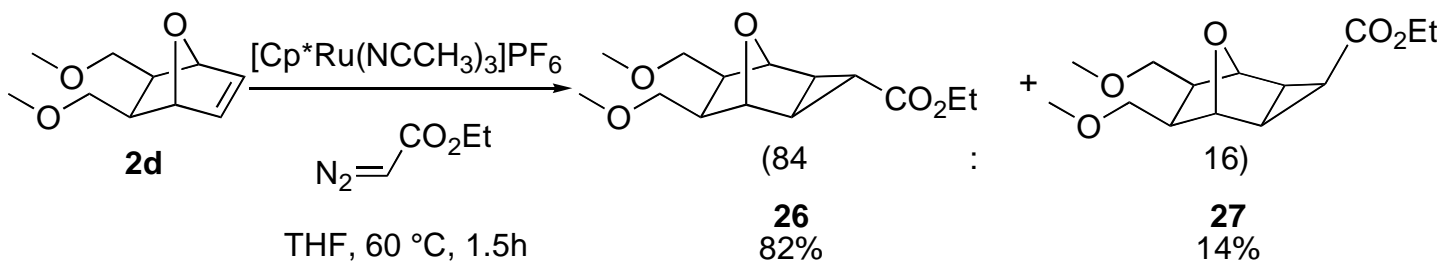

Cyclopropanes 26 and 27: Following the above general procedure (C) with alkene 2d (115.9 mg, $0.629 \mathrm{mmol})$, THF (0.5 mL), [Cp*Ru( $\left.\left(\mathrm{NCCH}_{3}\right)_{3}\right] \mathrm{PF}_{6}$ (34.1 mg, $0.0676 \mathrm{mmol}$ ), ethyl diazoacetate $(170 \mu \mathrm{L}, 1.64 \mathrm{mmol})$ and THF $(1.1 \mathrm{~mL})$. The addition was done over $30 \mathrm{~min}$ and the reaction mixture was stirred at $60{ }^{\circ} \mathrm{C}$ for $1.5 \mathrm{~h}$. The crude product was purified by column chromatography (gradient $\mathrm{Et}_{2} \mathrm{O}$ :hexanes $=1: 19$ to $1: 1$ ) to provide 26 (139.5 mg, $0.5161 \mathrm{mmol}, 82 \%)$ and 27 (23.8 mg, $0.0866 \mathrm{mmol}, 14 \%$ ) as colorless oils. Assignment of structures to these 2 epimers was based on the close analogies between these systems and those described by Sauers and co-workers, ${ }^{12}$ and Takahashi et al. ${ }^{13}$

26: $R_{f} 0.35$ (EtOAc:hexanes = 2:3); IR (neat) 2974, 2926, 2893, 2813, 1729, 1407, $1267 \mathrm{~cm}^{-1}$; ${ }^{1} \mathrm{H}$ NMR $\left(\mathrm{CDCl}_{3}, 400 \mathrm{MHz}\right) \delta 4.26$ (s, 2H), 4.04 (q, 2H, $\left.J=7.1 \mathrm{~Hz}\right), 3.24-3.28$ (m, 2H), 3.26 (s, 6H), 3.14-3.18 (m, 2H), 2.17-2.20 (m, 2H), 1.62-1.65 (m, 3H), 1.18 (t, 3H, $J=7.1 \mathrm{~Hz}){ }^{1} \mathrm{H}$ NMR ( $\left.\mathrm{C}_{6} \mathrm{D}_{6}, 400 \mathrm{MHz}\right) \delta 4.28$ (s, 2H), 3.93 (q, 2H, $J=7.1 \mathrm{~Hz}$ ), 3.08 (dd, 2H, $J=8.8,5.4$ Hz), 3.00-3.05 (m, 2H), 3.00 (s, 6H), 2.01-2.05 (m, 2H), 1.98 (t, 1H, J = 2.6 Hz), 1.57 (d, 2H, $J=2.6 \mathrm{~Hz}), 0.93$ (t, 3H, $J=7.1 \mathrm{~Hz}) ;{ }^{13} \mathrm{C} \mathrm{NMR}\left(\mathrm{APT}, \mathrm{CDCl}_{3}, 100 \mathrm{MHz}\right) \delta 172.9,78.2,70.5$, 60.5, 58.8, 45.8, 25.2, 16.4, 14.2. HRMS (EI) calcd. for $\mathrm{C}_{14} \mathrm{H}_{22} \mathrm{O}_{5}\left(\mathrm{M}^{+}\right)$: 270.1467; found: 270.1464 .

\footnotetext{
12 a) Sauers, R. R., Sonnet, P. E. Tetrahedron 1964, 20, 1029. b) Sauers, R. R.; Schlosberg, S. B.; Pfeffer, P. E. J. Org. Chem. 1968, 33, 2175.

${ }^{13}$ Matsushima, Y.; Kikuchi, H.; Uno, M.; Takahashi, S. Bull. Chem. Soc. Jpn. 1999, 72, 2475.
} 
26: $R_{f} 0.17$ (EtOAc:hexanes = 2:3); IR $\left(\mathrm{CH}_{2} \mathrm{Cl}_{2}\right)$ 2980, 2930, 2811, 1729, 1219, $1126 \mathrm{~cm}^{-1} ;{ }^{1} \mathrm{H}$ NMR (CDCl $3,400 \mathrm{MHz}) \delta 4.42$ (s, 2H), 4.09 (q, 2H, J = 7.1 Hz), 3.30-3.34 (m, 2H), 3.30 (s, 6H), 3.20-3.24 (m, 2H), 2.23-2.26 (m, 2H), 1.42-1.45 (m, 3H), 1.24 (t, 3H, $J=7.1 \mathrm{~Hz}$ ); ${ }^{1} \mathrm{H}$ NMR $\left(\mathrm{C}_{6} \mathrm{D}_{6}, 400 \mathrm{MHz}\right) \delta 4.60$ (s, 2H), 4.08 (q, 2H, $\left.J=7.1 \mathrm{~Hz}\right), 3.14$ (dd, 2H, $J=8.8,5.4$ Hz), 3.03-3.09 (m, 2H), 3.00 (s, 6H), 2.05-2.09 (m, 2H), 1.06 (t, 1H, J = 7.2 Hz), 1.02 (t, 3H, $J=7.1 \mathrm{~Hz}$ ), 0.87 (d, 2H, $J=7.2 \mathrm{~Hz}$ ); ${ }^{13} \mathrm{C}$ NMR (APT, $\left.\mathrm{CDCl}_{3}, 100 \mathrm{MHz}\right) \delta 170.6,78.5,70.6$, 60.3, 58.8, 46.3, 21.0, 19.5, 14.2. HRMS (EI) calcd. for $\mathrm{C}_{14} \mathrm{H}_{22} \mathrm{O}_{5}\left(\mathrm{M}^{+}\right)$: 270.1467; found: 270.1470 . 